\title{
Nanopore sequencing-based genome assembly and evolutionary genomics of circum-basmati rice
}

\author{
Jae Young Choi ${ }^{1 *} \mathbb{D}$, Zoe N. Lye', Simon C. Groen ${ }^{1}$, Xiaoguang Dai ${ }^{2}$, Priyesh Rughani², Sophie Zaaijer ${ }^{3}$, \\ Eoghan D. Harrington ${ }^{2}$, Sissel Juul ${ }^{2}$ and Michael D. Purugganan ${ }^{1,4^{*}}$
}

\begin{abstract}
Background: The circum-basmati group of cultivated Asian rice (Oryza sativa) contains many iconic varieties and is widespread in the Indian subcontinent. Despite its economic and cultural importance, a high-quality reference genome is currently lacking, and the group's evolutionary history is not fully resolved. To address these gaps, we use long-read nanopore sequencing and assemble the genomes of two circum-basmati rice varieties.

Results: We generate two high-quality, chromosome-level reference genomes that represent the 12 chromosomes of Oryza. The assemblies show a contig N50 of $6.32 \mathrm{Mb}$ and $10.53 \mathrm{Mb}$ for Basmati 334 and Dom Sufid, respectively. Using our highly contiguous assemblies, we characterize structural variations segregating across circum-basmati genomes. We discover repeat expansions not observed in japonica-the rice group most closely related to circumbasmati-as well as the presence and absence variants of over $20 \mathrm{Mb}$, one of which is a circum-basmati-specific deletion of a gene regulating awn length. We further detect strong evidence of admixture between the circumbasmati and circum-aus groups. This gene flow has its greatest effect on chromosome 10, causing both structural variation and single-nucleotide polymorphism to deviate from genome-wide history. Lastly, population genomic analysis of 78 circum-basmati varieties shows three major geographically structured genetic groups: Bhutan/Nepal, India/Bangladesh/Myanmar, and Iran/Pakistan.
\end{abstract}

Conclusion: The availability of high-quality reference genomes allows functional and evolutionary genomic analyses providing genome-wide evidence for gene flow between circum-aus and circum-basmati, describes the nature of circum-basmati structural variation, and reveals the presence/absence variation in this important and iconic rice variety group.

Keywords: Oryza sativa, Asian rice, Aromatic rice group, Domestication, Crop evolution, Nanopore sequencing, Aus, Basmati, Indica, Japonica, Admixture, Awnless, De novo genome assembly

\section{Background}

Oryza sativa or Asian rice is an agriculturally important crop that feeds one-half of the world's population [1] and supplies $20 \%$ of people's caloric intake (www.fao. org). Historically, O. sativa has been classified into two major variety groups, japonica and indica, based on morphometric differences and molecular markers [2,3]. These variety groups can be considered as subspecies, particularly

\footnotetext{
* Correspondence: jyc387@nyu.edu; mp132@nyu.edu

${ }^{1}$ Center for Genomics and Systems Biology, Department of Biology, New York University, New York, NY, USA

Full list of author information is available at the end of the article
}

given the presence of reproductive barriers between them [4]. Archaeobotanical remains suggest japonica rice was domesticated $\sim 9000$ years ago in the Yangtze Basin of China, while indica rice originated $\sim 4000$ years ago when domestication alleles were introduced from japonica into either O. nivara or a proto-indica in the Indian subcontinent [5]. More recently, two additional variety groups have been recognized that are genetically distinct from japonica and indica: the aus/circum-aus and aromatic/circum-basmati rice [6-8].

The rich genetic diversity of Asian rice is likely a result from a complex domestication process involving multiple

(c) The Author(s). 2020 Open Access This article is distributed under the terms of the Creative Commons Attribution 4.0 International License (http://creativecommons.org/licenses/by/4.0/), which permits unrestricted use, distribution, and reproduction in any medium, provided you give appropriate credit to the original author(s) and the source, provide a link to the Creative Commons license, and indicate if changes were made. The Creative Commons Public Domain Dedication waiver (http://creativecommons.org/publicdomain/zero/1.0/) applies to the data made available in this article, unless otherwise stated. 
wild progenitor populations and the exchange of important domestication alleles between $O$. sativa variety groups through gene flow [5, 7, 9-17]. Moreover, many agricultural traits within rice are variety group-specific [18-23], suggesting local adaptation to environments or cultural preferences have partially driven the diversification of rice varieties.

Arguably, the circum-basmati rice group has been the least studied among the four major variety groups, and it was only recently defined in more detail based on insights from genomic data [7]. Among its members, the group boasts the iconic basmati rice (sensu stricto) from southern Asia and the sadri rice from Iran [6]. Many, but not all, circum-basmati varieties are characterized by distinct and highly desirable fragrance and texture [24]. Nearly all fragrant circum-basmati varieties possess a loss-of-function mutation in the $B A D H 2$ gene that has its origins in ancestral japonica haplotypes, suggesting that an introgression between circum-basmati and japonica may have led to fragrant basmati rice $[21,25,26]$. Genome-wide polymorphism analysis of a smaller array of circum-basmati rice cultivars shows close association with japonica varieties [7, 16, 27], providing evidence that at least part of the genomic make-up of circumbasmati rice may indeed be traced back to japonica.

Whole-genome sequences are an important resource for evolutionary geneticists studying plant domestication, as well as breeders aiming to improve crop varieties. Single-molecule sequencing regularly produces sequencing reads in the range of kilobases $(\mathrm{kb})$ [28]. This is particularly helpful for assembling plant genomes, which are often highly repetitive and heterozygous, and commonly underwent at least one round of polyploidization in the past [29-31]. The Oryza sativa genome, with a relatively modest size of $\sim 400 \mathrm{Mb}$, was the first crop genome sequence assembled [29], and there has been much progress in generating de novo genome assemblies for other members of the genus Oryza. Currently, there are assemblies for nine wild species (Leersia perrieri [outgroup], O. barthii, O. brachyantha, O. glumaepatula, O. longistaminata, O. meridionalis, O. nivara, O. punctata, and O. rufipogon) and two domesticated species $(O$. glaberrima and O. sativa) [32-37].

Within domesticated Asian rice (O. sativa), genome assemblies are available for cultivars in most variety groups [32, 33, 38-42]. However, several of these reference assemblies are based on short-read sequencing data and show higher levels of incompleteness compared to assemblies generated from long-read sequences [40, 41]. Nevertheless, these de novo genome assemblies have been critical in revealing genomic variation (e.g., variations in genome structure and repetitive DNA, and de novo species- or population-specific genes) that were otherwise missed from analyzing a single reference genome. Recently, a genome assembly based on short-read sequencing data was generated for basmati rice [42]. Not only were there missing sequences in this assembly, it was also generated from DNA of an elite basmati breeding line. Such modern cultivars are not the best foundations for domesticationrelated analyses due to higher levels of introgression from other rice populations during modern breeding.

Here, we report the de novo sequencing and assembly of the landraces (traditional varieties) Basmati 334 [21, 43, 44] and Dom Sufid $[21,24,45,46]$ using the long-read nanopore sequencing platform of Oxford Nanopore Technologies [47]. Basmati 334 is from Pakistan, evolved in a rainfed lowland environment and is known to be drought tolerant at the seedling and reproductive stages [44]. It also possesses several broad-spectrum bacterial blight resistance alleles [48, 49], making Basmati 334 desirable for breeding resilience into modern basmati cultivars [49, 50]. Dom Sufid is an Iranian sadri cultivar that, like other sadri and basmati (sensu stricto) varieties, is among the most expensive varieties currently available in the market [24]. It has desirable characteristics such as aromaticity and grain elongation during cooking, although it is susceptible to disease and abiotic stress [24, 51]. Because of their special characteristics, both Basmati 334 and Dom Sufid are used in elite rice breeding programs to create high yielding and resilient aromatic rice varieties [24, 44-46, 50].

Based on long reads from nanopore sequencing, our genome assemblies have high quality, contiguity, and genic completeness, making them comparable in quality to assemblies associated with key rice reference genomes. We used our circum-basmati genome assemblies to characterize genomic variation existing within this important rice variety group, and analyze domesticationrelated and other evolutionary processes that shaped this variation. Our circum-basmati rice genome assemblies will be valuable complements to the available assemblies for other rice cultivars, unlocking important genomic variation for rice crop improvement.

\section{Results \\ Nanopore sequencing of basmati and sadri rice}

Using Oxford Nanopore Technologies' long-read sequencing platform, we sequenced the genomes of the circum-basmati landraces Basmati 334 (basmati sensu stricto) and Dom Sufid (sadri). We called 1,372,950 reads constituting a total of $29.2 \mathrm{~Gb}$ for Basmati 334 and $1,183,159$ reads constituting a total of $24.2 \mathrm{~Gb}$ for Dom Sufid (Table 1). For both samples, the median read length was $>17 \mathrm{~kb}$, the read length N50 was $>33 \mathrm{~kb}$, and the median quality score per read was $\sim 11$.

\section{De novo assembly of the Basmati 334 and Dom Sufid rice genomes}

Incorporating only those reads that had a mean quality score of $>8$ and read lengths of $>8 \mathrm{~kb}$, we used a total 
Table 1 Summary of nanopore sequencing read data

\begin{tabular}{llllll}
\hline Flowcell & Number of reads & Median read length & Read length N50 & Median quality score (QS) & Total bases \\
\hline & Basmati 334 & & & & 11.27 \\
FAK30515 & 288,473 & 19,905 & 36,743 & 11.19 & $6,843,069,570$ \\
FAK30732 & 306,247 & 18,792 & 30,974 & 11.07 & $6,341,851,953$ \\
FAK30522 & 228,191 & 17,366 & 36,456 & 11.35 & $4,816,938,523$ \\
FAK27872 & 244,606 & 18,335 & 31,267 & 11.43 & $5,045,781,146$ \\
FAK27919 & 305,433 & 18,087 & 30,727 & 11.27 & $6,191,306,294$ \\
All & $1,372,950$ & 18,576 & 33,005 & & $29,238,947,486$ \\
& Dom Sufid & & & 11.34 & 11.30 \\
FAK30464 & 300,290 & 18,477 & 37,754 & 10.96 & $6,681,819,859$ \\
FAK30582 & 258,584 & 17,641 & 34,213 & 10.99 & $5,353,774,444$ \\
FAK28890 & 330,924 & 16,756 & 34,033 & 11.14 & $6,553,200,184$ \\
FAK30064 & 293,361 & 16,178 & 32,835 & $3,618,557,776$ \\
All & $1,183,159$ & 17,237 & 34,728 & $24,207,352,263$ \\
\hline
\end{tabular}

of 1,076,192 reads and 902,040 reads for the Basmati 334 and Dom Sufid genome assemblies, which resulted in a genome coverage of $\sim 62 \times$ and $\sim 51 \times$, respectively (Table 2). We polished the genome assemblies with both nanopore and short Illumina sequencing reads. The final, polished genome assemblies spanned $386.5 \mathrm{Mb}$ across 188 contigs for Basmati 334 and $383.6 \mathrm{Mb}$ across 116 contigs for Dom Sufid. The genome assemblies had high contiguity, with a contig N50 of $6.32 \mathrm{Mb}$ and 10.53 Mb for Basmati 334 and Dom Sufid, respectively. Our genome assemblies recovered more than $97 \%$ of the 1440 BUSCO [52] embryophyte gene groups, which is comparable to the BUSCO statistics for the japonica Nipponbare [33] (98.4\%) and indica R498 reference genomes [41] (98.0\%). This is an improvement from the

Table 2 Summary of the circum-basmati rice genome assemblies

\begin{tabular}{lll}
\hline & Basmati 334 & Dom Sufid \\
\hline Genome coverage & $62.5 \times$ & $51.4 \times$ \\
Number of contigs & 188 & 116 \\
Total number of bases in contigs & $386,555,741$ & $383,636,250$ \\
Total number of bases scaffolded & $386,050,525$ & $383,245,802$ \\
Contig N50 length & $6.32 \mathrm{Mb}$ & $10.53 \mathrm{Mb}$ \\
Contig L50 & 20 & 13 \\
Total contigs > 50 kbp & 159 & 104 \\
Maximum contig length & $17.04 \mathrm{Mb}$ & $26.82 \mathrm{Mb}$ \\
BUSCO gene completion (assembly) & $97.6 \%$ & $97.0 \%$ \\
GC content & $43.6 \%$ & $43.7 \%$ \\
Repeat content & $44.4 \%$ & $44.2 \%$ \\
Number of annotated genes & 41,270 & 38,329 \\
BUSCO gene completion (annotation) & $95.4 \%$ & $93.6 \%$ \\
\hline
\end{tabular}

currently available genome assembly of basmati variety GP295-1 [42], which was generated from Illumina shortread sequencing data and has a contig N50 of $44.4 \mathrm{~kb}$ with 50,786 assembled contigs.

We examined coding sequences of our circum-basmati genomes by conducting gene annotation using published rice gene models and the MAKER gene annotation pipeline $[52,53]$. A total of 41,270 genes were annotated for the Basmati 334 genome, and 38,329 for the Dom Sufid genome. BUSCO gene completion analysis [52] indicated that $95.4 \%$ and $93.6 \%$ of the 3278 single-copy genes from the liliopsida gene dataset were found in the Basmati 334 and Dom Sufid gene annotations, respectively.

\section{Whole-genome comparison to other rice variety group genomes}

We aligned our draft genome assemblies to the japonica Nipponbare reference genome sequence [33], which represents one of the highest quality reference genome sequences (Fig. 1a). Between the Nipponbare, Basmati 334 and Dom Sufid genomes, high levels of macrosynteny were evident across the japonica chromosomes. Specifically, we observed little large-scale structural variation between Basmati 334 and Dom Sufid contigs and the japonica genome. A noticeable exception was an apparent inversion in the circum-basmati genome assemblies at chromosome 6 between positions 12.5 and 18.7 Mb (Nipponbare coordinates), corresponding to the pericentromeric region [54]. Interestingly, the same region showed an inversion between the Nipponbare and indica R498 reference genomes [41], whereas in the circum-aus N22 cultivar no inversions are observed (Additional file 1: Figure S1). While the entire region was inverted in R498, the inversion positions were disjoint in Basmati 334 and Dom Sufid, apparently 


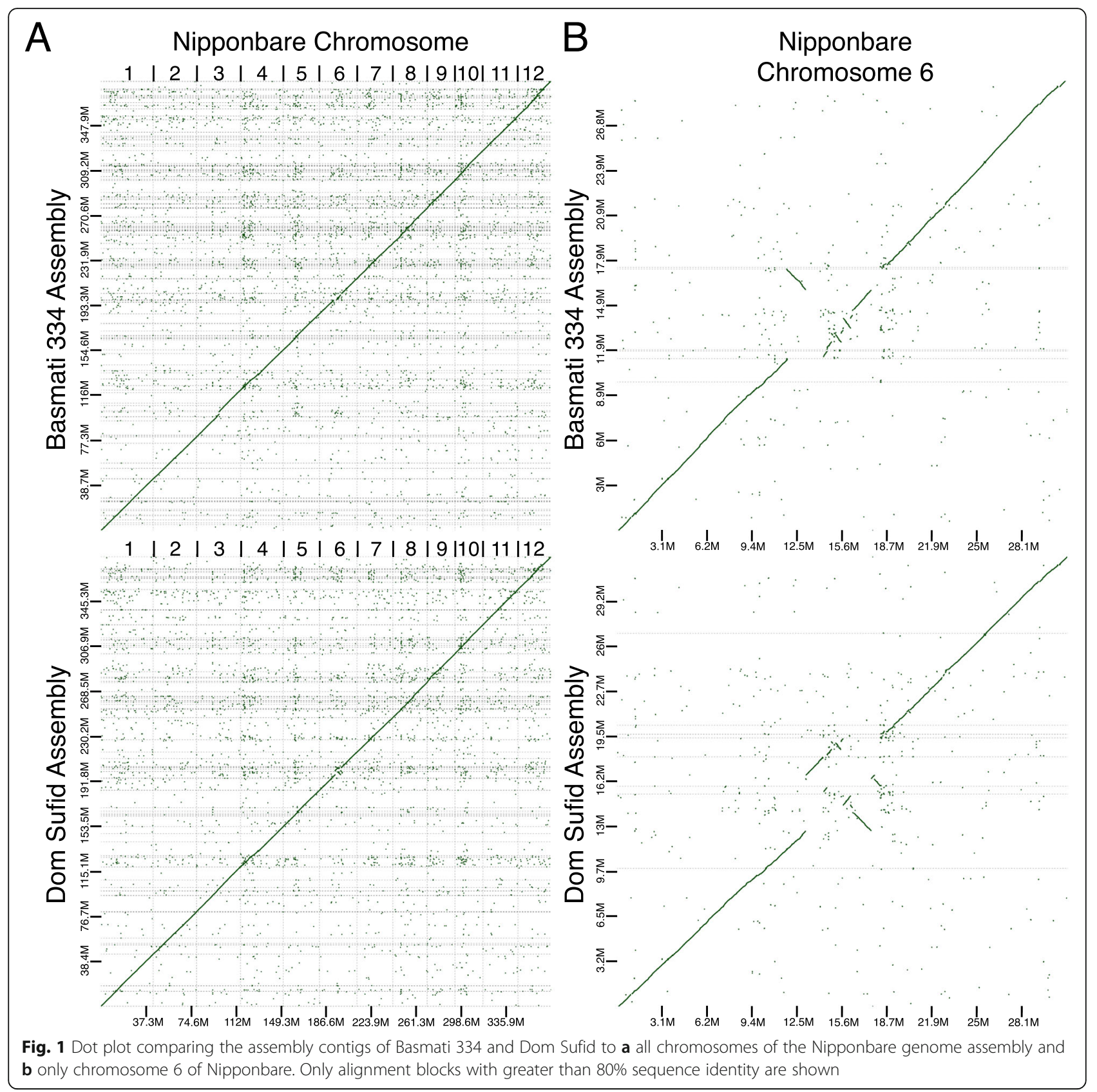

occurring in multiple regions of the pericentromere. We independently verified the inversions by aligning raw nanopore sequencing reads to the Nipponbare reference genome using the long-read-aware aligner $n g m l r$ [55], and the structural variation detection program sniffles [55]. Sniffles detected several inversions, including a large inversion between positions 13.1 and 17.7 $\mathrm{Mb}$ and between 18.18 and $18.23 \mathrm{Mb}$, with several smaller inversions located within the largest inversion (Additional file 2: Table S1).

Because of high macro-synteny with japonica (Fig. 1a), we ordered and oriented the contigs of the Basmati 334 and Dom Sufid assemblies using a reference genomebased scaffolding approach [56]. For both Basmati 334 and Dom Sufid, over $99.9 \%$ of the assembled genomic contigs were anchored to the Nipponbare reference genome (Table 2). The scaffolded circum-basmati chromosomes were similar in size to those in reference genomes for cultivars in other rice variety groups (Nipponbare [33], the circum-aus variety N22 [37], and the indica varieties IR8 [37] and R498 [41]) that were sequenced, assembled, and scaffolded to near completion (Table 3).

Next, we assessed the assembly quality of the circumbasmati genomes by contrasting them against available 
Table 3 Comparison of assembled chromosome sizes for cultivars across variety groups

\begin{tabular}{lllllll}
\hline Chromosome & Basmati 334 & Dom Sufid & Nipponbare & N22 & IR8 & R498 \\
\hline 1 & $44,411,451$ & $44,306,286$ & $43,270,923$ & $44,711,178$ & $44,746,683$ & $44,361,539$ \\
2 & $35,924,761$ & $36,365,206$ & $35,937,250$ & $38,372,633$ & $37,475,564$ & $37,764,328$ \\
3 & $38,133,813$ & $36,413,819$ & $36,762,248$ & $39,065,119$ & $39,691,490$ \\
4 & $40,305,655$ & $34,714,597$ & $35,502,694$ & $33,558,078$ & $35,713,470$ & $35,849,732$ \\
5 & $34,905,232$ & $31,017,353$ & $29,958,434$ & $28,792,057$ & $31,269,760$ & $31,237,231$ \\
6 & $30,669,872$ & $32,412,977$ & $31,248,787$ & $29,772,976$ & $32,072,649$ & $32,465,040$ \\
7 & $29,982,228$ & $29,511,326$ & $29,697,621$ & $29,936,233$ & $30,380,234$ & $30,277,827$ \\
8 & $30,410,531$ & $29,962,976$ & $28,443,022$ & $25,527,801$ & $30,236,384$ & $29,952,003$ \\
9 & $29,921,941$ & $23,970,096$ & $23,012,720$ & $22,277,206$ & $24,243,884$ & $24,760,661$ \\
10 & $24,050,083$ & $24,989,786$ & $23,207,287$ & $20,972,683$ & $25,246,678$ & $25,582,588$ \\
11 & $25,596,481$ & $29,949,236$ & $29,021,106$ & $29,032,419$ & $32,337,678$ & $31,778,392$ \\
12 & $29,979,012$ & $27,912,150$ & $27,531,856$ & $22,563,585$ & $25,963,606$ & $26,601,357$ \\
Total & $29,893,278$ & $383,245,802$ & $373,245,519$ & $362,279,097$ & $388,751,709$ & $390,322,188$ \\
\hline
\end{tabular}

de novo-assembled genomes within the Asian rice complex (see the "Materials and methods" section for a complete list of genomes). We generated a multigenome alignment to the Nipponbare genome, which we chose as the reference since its assembly and gene annotation is a product of years of community-based efforts $[33,57,58]$. To infer the quality of the gene regions in each of the genome assemblies, we used the multigenome alignment to extract the coding DNA sequence of each Nipponbare gene and its orthologous regions from each non-japonica genome. The orthologous genes were counted for missing DNA sequences ("N" sequences) and gaps to estimate the percent of Nipponbare genes covered. For all genomes, the majority of Nipponbare genes had a near-zero proportion of sites that were missing in the orthologous non-Nipponbare genes (Additional file 1: Figure S2). The missing proportions of Nipponbare-orthologous genes within the Basmati 334 and Dom Sufid genomes were comparable to those for genomes that had higher assembly contiguity $[37,40,41]$.

Focusing on the previously sequenced basmati GP295-1 genome [42], our newly assembled circum-basmati genomes had noticeably lower proportions of missing genes (Additional file 1: Figure S2). Furthermore, over 96\% of base pairs across the Nipponbare genome were alignable against the Basmati 334 (total of 359,557,873 bp [96.33\%] of Nipponbare genome) or Dom Sufid (total of 359,819, 239 bp [96.40\%] of Nipponbare genome) assemblies, while only $194,464,958 \mathrm{bp}(52.1 \%)$ of the Nipponbare genome were alignable against the GP295-1 assembly.

We then counted the single-nucleotide and insertion/ deletion (indel, up to $\sim 60 \mathrm{bp}$ ) differences between the circum-basmati and Nipponbare assemblies to assess the overall quality of our newly assembled genomes. To avoid analyzing differences across unconstrained repeat regions, we specifically examined regions where there were 20 exact base-pair matches flanking a site that had a single-nucleotide or indel difference between the circum-basmati and Nipponbare genomes. In the GP2951 genome, there were $334,500(0.17 \%)$ single-nucleotide differences and 44,609 (0.023\%) indels compared to the Nipponbare genome. Our newly assembled genomes had similar proportions of single-nucleotide differences with the Nipponbare genome, where the Basmati 334 genome had 780,735 (0.22\%) differences and the Dom Sufid genome had 731,426 (0.20\%). For indels, the Basmati 334 genome had comparable proportions of differences with 104,282 (0.029\%) variants, but the Dom Sufid genome had higher proportions with $222,813(0.062 \%)$ variants. In sum, our draft circum-basmati genomes had high contiguity and completeness as evidenced by assembly to the chromosome level and comparison to the Nipponbare genome. In addition, our genome assemblies were comparable to the Illumina sequence-generated GP295-1 genome for the proportion of genomic differences with the Nipponbare genome, suggesting they had high quality and accuracy as well.

Our circum-basmati genome assemblies should also be of sufficiently high quality for detailed gene-level analysis. For instance, a hallmark of many circum-basmati rice is aromaticity, and a previous study had determined that Dom Sufid, but not Basmati 334, is a fragrant variety [21]. We examined the two genomes to verify the presence or absence of the mutations associated with fragrance. There are multiple different loss-of-function mutations in the $B A D H 2$ gene that cause rice varieties to be fragrant $[21,25,26]$, but the majority of fragrant rice carry a deletion of 8 nucleotides at position chr8:20, $382,861-20,382,868$ of the Nipponbare genome assembly 
(version Os-Nipponbare-Reference-IRGSP-1.0). Using the genome alignment, we extracted the $B A D H 2$ sequence region to compare the gene sequence of the non-fragrant Nipponbare to that of Basmati 334 and Dom Sufid. Consistent with previous observations [21], we found that the genome of the non-fragrant Basmati 334 did not carry the deletion and contained the wildtype $B A D H 2$ haplotype observed in Nipponbare. The genome of the fragrant Dom Sufid, on the other hand, carried the 8-bp deletion, as well as the 3 singlenucleotide polymorphisms flanking the deletion. This illustrates that the Basmati 334 and Dom Sufid genomes are accurate enough for gene-level analysis.

\section{Circum-basmati gene analysis}

Our annotation identified $\sim 40,000$ coding sequences in the circum-basmati assemblies. We examined population frequencies of the annotated gene models across a circum-basmati population dataset to filter out misannotated gene models or genes at very low frequency in a population. We obtained Illumina sequencing reads from varieties included in the 3K Rice Genome Project [7] and sequenced additional varieties to analyze a total of 78 circum-basmati cultivars (see Additional file 2: Table S2 for a list of varieties). The Illumina sequencing reads were aligned to the circum-basmati genomes, and if the average coverage of a genic region was $<0.05 \times$ for an individual, this gene was called as a deletion in that variety. Since we used a low threshold for calling a deletion, the genome-wide sequencing coverage of a variety did not influence the number of gene deletions detected (Additional file 1: Figure S3). Results showed that gene deletions were indeed rare across the circum-basmati population (Fig. 2a), consistent with their probable deleterious nature. We found that 31,565 genes $(76.5 \%)$ in Basmati 334 and 29,832 genes (77.8\%) in the Dom Sufid genomes did not have a deletion across the population (see Additional file 2: Table S3 for a list of genes).

There were 517 gene models from Basmati 334 and 431 gene models from Dom Sufid that had a deletion frequency of $\geq 0.3$ (see Additional file 2: Table S4 for a list of genes). These gene models with high deletion frequencies were not considered further in this analysis. The rest were compared against the circum-aus $\mathrm{N} 22$, indica $\mathrm{R} 498$, and japonica Nipponbare gene models to determine their orthogroup status (Fig. 2b; see Additional file 2: Table S5 for a list of genes and their orthogroup status), which are sets of genes that are orthologs and recent paralogs of each other [59].

The most frequent orthogroup class observed was for groups in which every rice variety group has at least one gene member. There were 13,894 orthogroups within this class, consisting of 17,361 genes from N22, 18,302 genes from Basmati 334, 17,936 genes from Dom Sufid,
17,553 genes from R498, and 18,351 genes from Nipponbare. This orthogroup class likely represents the set of core genes of $O$. sativa [42]. The second-highest orthogroup class observed was for groups with genes that were uniquely found in both circum-basmati genomes (3802 orthogroups). These genes represent those restricted to the circum-basmati group.

In comparison to genes in other rice variety groups, the circum-basmati genes shared the highest number of orthogroups with circum-aus (2648 orthogroups), followed by japonica (1378 orthogroups), while sharing the lowest number of orthogroups with indica (663 orthogroups). In fact, genes from indica variety R498 had the lowest number assigned to an orthogroup (Fig. $2 \mathrm{~b}$ inset table), suggesting this genome had more unique genes, i.e., without ortho$\operatorname{logs} /$ paralogs to genes in other rice variety groups.

\section{Genome-wide presence/absence variation within the circum-basmati genomes}

Our assembled circum-basmati genomes were $>10 \mathrm{Mb}$ longer than the Nipponbare genome, but individual chromosomes showed different relative lengths (Table 3) suggesting a considerable number of presence/absence variants (PAVs) between the genomes. We examined the PAVs between the circum-basmati and Nipponbare genomes using two different computational packages: (i) sniffles, which uses raw nanopore reads aligned to a reference genome to call PAVs, and (ii) assemblytics [60], which aligns the genome assemblies to each other and calls PAVs. The results showed that, while the total number of PAVs called by sniffles and assemblytics were similar, only $\sim 36 \%$ of PAVs had overlapping positions (Table 4). In addition, the combined total size of PAVs was larger for predictions made by sniffles compared to those by assemblytics. For subsequent analysis, we focused on PAVs that were called by both methods.

The distribution of PAV sizes indicated that large PAVs were rare across the circum-basmati genomes, while PAVs $<500$ bps in size were the most common (Fig. 3a). Within smaller-sized PAVs those in the 200-500-bp size range showed a peak in abundance. A closer examination revealed that sequence positions of more than $75 \%$ of these 200-500-bp-sized PAVs overlapped with transposable element coordinates in the circum-basmati genomes (Additional file 2: Table S6). A previous study based on short-read Illumina sequencing data reported a similar enrichment of short repetitive elements such as the long terminal repeats (LTRs) of retrotransposons, Tc1/mariner elements, and mPing elements among PAVs in this size range [61].

PAVs shorter than 200 bps also overlapped with repetitive sequence positions in the circum-basmati genomes, but the relative abundance of each repeat type differed among insertion and deletion variants. Insertions in the 


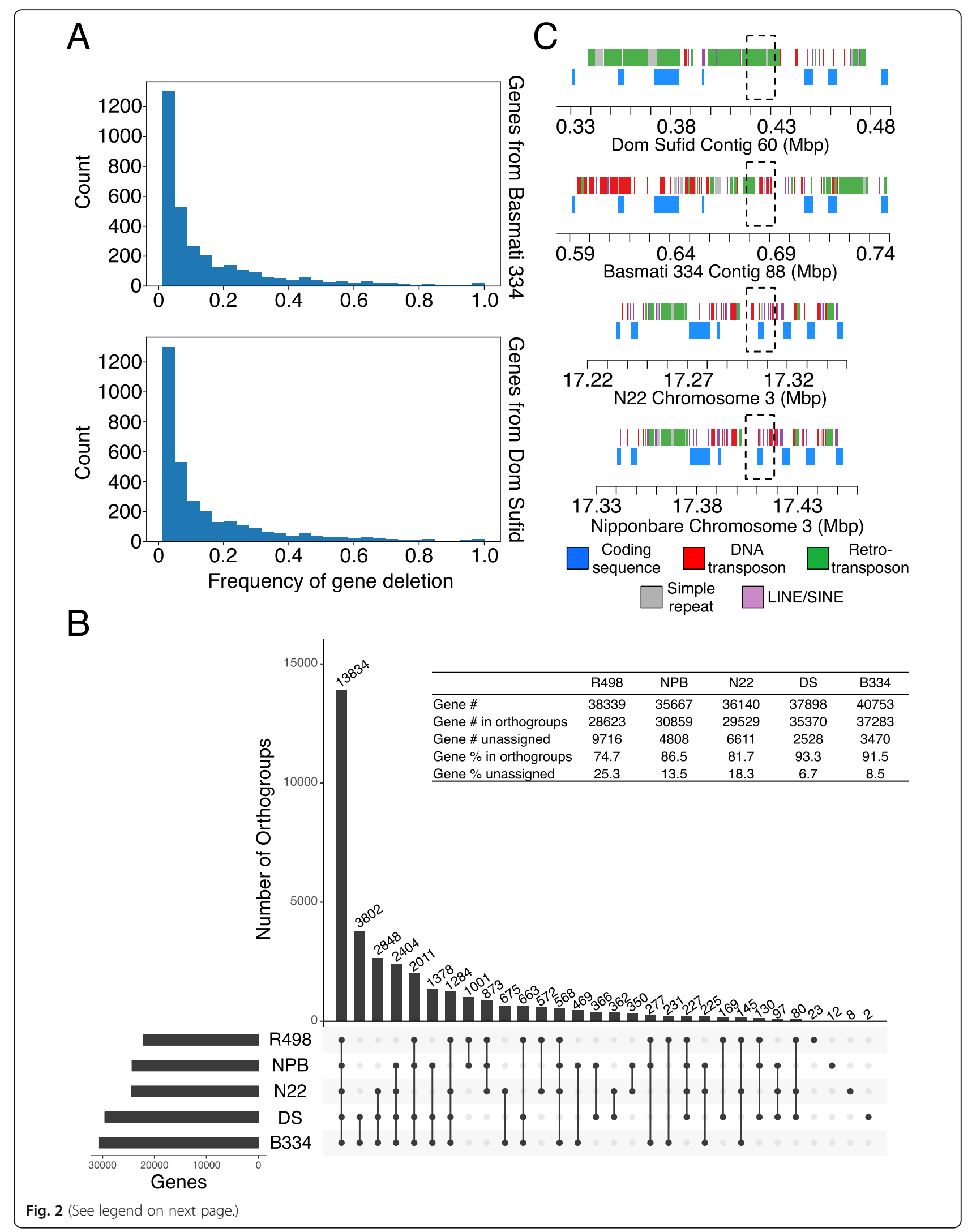


(See figure on previous page.)

Fig. 2 Circum-basmati gene sequence evolution. a The deletion frequency of genes annotated from the Basmati 334 and Dom Sufid genomes. Frequency was estimated from sequencing data on a population of 78 circum-basmati varieties. $\mathbf{b}$ Groups of orthologous and paralogous genes (i.e., orthogroups) identified in the reference genomes of circum-aus N22, japonica Nipponbare (NPB), and indica R498, as well as the circum-basmati genome assemblies Basmati 334 (B334) and Dom Sufid (DS) of this study. $\mathbf{c}$ Visualization of the genomic region orthologous to the Nipponbare gene Os03g0418600 (Awn3-1) in the N22, Basmati 334, and Dom Sufid genomes. Regions orthologous to Awn3-1 are indicated with a dotted box

Basmati 334 and Dom Sufid genomes had a higher relative abundance of simple sequence repeats (i.e., microsatellites) compared to deletions (Additional file 2: Table S6). These inserted simple sequence repeats were highly enriched for (AT) $)_{n}$ dinucleotide repeats, which in Basmati 334 accounted for 66,624 bps out of a total of $72,436 \mathrm{bps}(92.0 \%)$ of simple sequence repeats, and for Dom Sufid 56,032 bps out of a total of 63,127 bps $(88.8 \%)$.

Between the Basmati 334 and Dom Sufid genomes, $\sim 45 \%$ of PAVs had overlapping genome coordinates (Fig. $3 \mathrm{~b}$ ) suggesting that variety-specific insertion and deletion polymorphisms were common. We plotted PAVs for each of our circum-basmati genomes to visualize their distribution (Fig. 3c). Chromosomespecific differences in the distribution of PAVs were seen for each circum-basmati genome: in Basmati 334, for example, chromosome 1 had the lowest density of PAVs, while in Dom Sufid this was the case for chromosome 2 (Additional file 1: Figure S4). On the other hand, both genomes showed significantly higher densities of PAVs on chromosome 10 (Tukey's range test $p<0.05)$. This suggested that, compared to Nipponbare, chromosome 10 was the most differentiated in terms of insertion and deletion variations in both of our circum-basmati genomes.

\section{Evolution of circum-basmati rice group-specific gene presence and absence variation}

The proportion of repeat sequences found within the larger-sized PAVs (i.e., those $>2 \mathrm{~kb}$ ) was high, where

Table 4 Comparison of presence/absence variation called by two different computational packages

\begin{tabular}{llll}
\hline & Sniffles & Assemblytics & Overlap \\
\hline Basmati 334 & & & \\
Deletion counts & 11,989 & 11,247 & 4051 \\
Deleted base-pairs & $43,768,763$ & $29,048,238$ & $19,328,854$ \\
Insertion counts & 11,447 & 12,161 & 3734 \\
Inserted base-pairs & $19,650,518$ & $14,498,550$ & $5,783,551$ \\
Dom Sufid & & & \\
Deletion counts & 9901 & 10,115 & 3649 \\
Deleted base-pairs & $36,600,114$ & $26,128,143$ & $17,274,967$ \\
Insertion counts & 9834 & 11,134 & 3340 \\
Inserted base-pairs & $16,527,995$ & $12,902,410$ & $5,160,503$ \\
\hline
\end{tabular}

between 84 and $98 \%$ of large PAVs contained transposable element-related sequences (Additional file 2: Table S6). Regardless, these larger PAVs also involved loss or gain of coding sequences. For instance, gene ontology analysis of domesticated rice gene orthogroups showed enrichment for genes related to electron transporter activity among both circum-basmati-specific gene losses and gains (see Additional file 2: Table S7 for gene ontology results for circum-basmati-specific gene losses and Additional file 2: Table S8 for gene ontology results for circum-basmati-specific gene gains).

Many of these genic PAVs could have been important during the rice domestication process [11]. Gene deletions, in particular, are more likely to have a functional consequence than single-nucleotide polymorphisms or short indels and may underlie drastic phenotypic variation. In the context of crop domestication and diversification, this could have led to desirable phenotypes in human-created agricultural environments. For instance, several domestication phenotypes in rice are known to be caused by gene deletions [35, 62-66].

There were 873 gene orthogroups for which neither of the circum-basmati genomes had a gene member, but for which genomes for all three other rice variety groups (N22, Nipponbare, and R498) had at least one gene member. Among these, there were 545 orthogroups for which N22, Nipponbare, and R498 each had a single-copy gene member, suggesting that the deletion of these genes in both the Basmati 334 and Dom Sufid genomes could have had a major effect in circum-basmati. We aligned Illumina sequencing data from our circum-basmati population dataset to the japonica Nipponbare genome and calculated deletion frequencies of Nipponbare genes that belonged to the 545 orthogroups (see Additional file 2: Table S9 for gene deletion frequencies in the circum-basmati population for the Nipponbare genes that are missing in Basmati 334 and Dom Sufid). The vast majority of these Nipponbare genes (509 orthogroups or 93.4\%) were entirely absent in the circum-basmati population, further indicating that these were circum-basmati-specific gene deletions fixed within this variety group.

One of the genes specifically deleted in circumbasmati rice varieties was Awn3-1 (Os03g0418600), which was identified in a previous study as associated with altered awn length in japonica rice [67]. Reduced awn length is an important domestication trait that was selected for ease of harvesting and storing rice seeds 


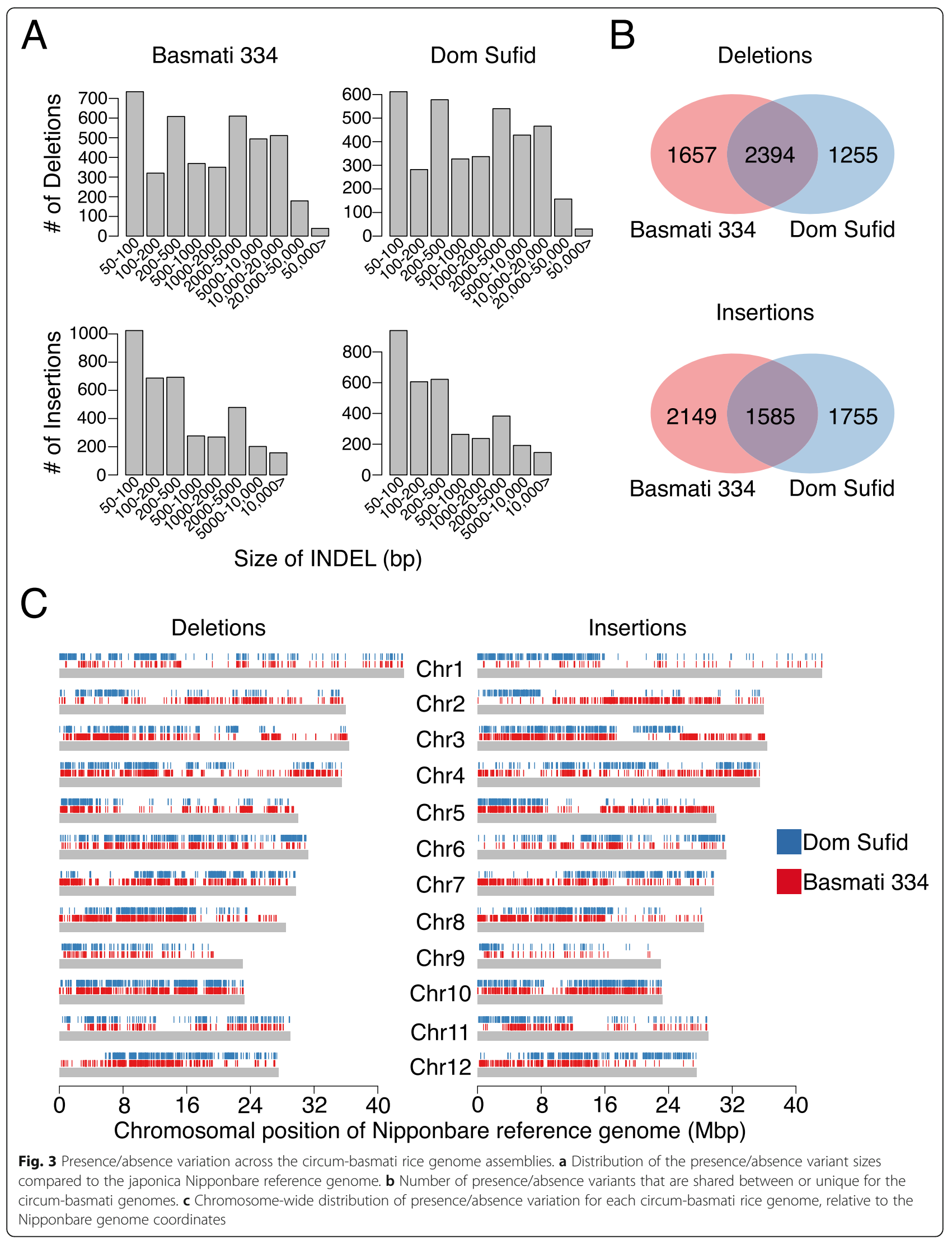


[68]. This gene was missing in both circum-basmati genomes, and no region could be aligned to the Nipponbare Awn3-1 genic region (Fig. 2c). Instead of the Awn31 coding sequence, this genomic region contained an excess of transposable element sequences, suggesting an accumulation of repetitive DNA may have been involved in this gene's deletion. The flanking arms upstream and downstream of Os03g0418600 were annotated in both circum-basmati genomes and were syntenic to the regions in both Nipponbare and N22. These flanking arms, however, were also accumulating transposable element sequences, indicating that this entire genomic region may be degenerating in both circum-basmati rice genomes.

We then examined the deletion status for other genes involved in the domestication of $O$. sativa. We focused on the genes that were previously implicated to be involved in the initial domestication phase of rice [11] where the genes were selected during the transformation of a wild rice into a domesticated rice-i.e., $R c$ (Os07g0211500) [19], Bh4 (Os04g0460200) [69], PROG1 (Os07g0153600) [70], OsC1 (Os06g0205100) [71], Sh4 (Os04g0670900) [72], GS3 (Os03g0407400) [73], qSH1 (Os01g0848400) [20], and qSW5 (Os05g0187500) [62]. Our aim was to draw inferences on whether the domestication history of circum-basmati rice may have differed from that of the other rice subpopulations. Results showed none of these genes were deleted in the circum-basmati population (Additional file 2: Table S8). This suggests that unlike the domestication process of domesticated African rice $(O$. glaberrima [74]), gene deletions were not a major contributor during the initial domestication phase of circumbasmati rice. Its likely many of the gene deletions that were selected during the domestication of the circumbasmati rice occurred during the cultivation period [11], when culinary or cultural preferences have selected for unique circum-basmati specific traits.

\section{Repetitive DNA and retrotransposon dynamics in the circum-basmati genomes}

Repetitive DNA makes up more than $44 \%$ of the Basmati 334 and Dom Sufid genome assemblies (Table 2). Consistent with genomes of other plant species [75], the repetitive DNA was largely composed of Class I retrotransposons, followed by Class II DNA transposons (Fig. 4a). In total, 171.1 Mb was annotated as repetitive for Basmati 334 and 169.5 Mb for Dom Sufid. The amount of repetitive DNA in the circum-basmati genomes was higher than that in the Nipponbare $(160.6 \mathrm{Mb})$ and N22 genomes (152.1 $\mathrm{Mb}$ ), but lower than that in the indica R498 (175.9 $\mathrm{Mb})$ and IR8 $(176.0 \mathrm{Mb})$ genomes. These differences in the total amount of repetitive DNA were similar to overall genome assembly size differences (Table 3), indicating that variation in repeat DNA accumulation is largely driving genome size differences in rice [76].
We focused our attention on retrotransposons, which made up the majority of the rice repetitive DNA landscape (Fig. 4a). Using LTRharvest [77, 78], we identified and de novo-annotated LTR retrotransposons in the circumbasmati genomes. LTRharvest annotated 5170 and 5150 candidate LTR retrotransposons in Basmati 334 and Dom Sufid, respectively (Additional file 2: Tables S10 and S11). Of these, 4180 retrotransposons $(80.9 \%$ of all candidate LTR retrotransposons) in Basmati 334 and 4228 (82.1\%) in Dom Sufid were classified as LTR retrotransposons by RepeatMasker's RepeatClassifer tool (http://www.repeat masker.org). Most LTR retrotransposons were from the gypsy and copia superfamilies $[79,80]$, which made up 77.1\% (3225 gypsy elements) and 21.9\% (915 copia elements) of LTR retrotransposons in the Basmati 334 genome, and 76.4\% (3231 gypsy elements) and 22.8\% (962 copia elements) of LTR retrotransposons in the Dom Sufid genome, respectively. Comparison of LTR retrotransposon content among reference genomes from different rice variety groups (Additional file 1: Figure S5) revealed that genomes assembled to near completion (i.e., Nipponbare, N22, Basmati 334, Dom Sufid, and indica varieties IR8 and R498, as well as MH63 and ZS97 [40]) had higher numbers of annotated retrotransposons than genomes generated from short-read sequencing data (GP295-1, circum-aus varieties DJ123 [38] and Kasalath [39], and indica variety IR64 [38]), suggesting genome assemblies from short-read sequencing data may be missing certain repetitive DNA regions.

Due to the proliferation mechanism of LTR transposons, the DNA divergence of an LTR sequence can be used to approximate the insertion time for an LTR retrotransposon [81]. Compared to other rice reference genomes, the insertion times for the Basmati 334 and Dom Sufid LTR retrotransposons were most similar to those observed for elements in the circum-aus N22 genome (Additional file 1: Figure S5). Within our circum-basmati assemblies, the gypsy superfamily elements had a younger average insertion time ( 2.2 million years ago) than elements of the copia superfamily ( $\sim 2.7$ million years ago; Fig. $4 \mathrm{~b})$.

Concentrating on gypsy and copia elements with the rve (integrase; Pfam ID: PF00665) gene, we examined the evolutionary dynamics of these LTR retrotransposons by reconstructing their phylogenetic relationships across reference genomes for the four domesticated rice variety groups (N22, Basmati 334, Dom Sufid, R498, IR8, and Nipponbare), and the two wild rice species ( $O$. nivara and O. rufipogon; Fig. 4c). The retrotransposons grouped into distinct phylogenetic clades, which likely reflect repeats belonging to the same family or subfamily [82]. The majority of phylogenetic clades displayed short external and long internal branches, consistent with rapid recent bursts of transposition observed across various rice LTR retrotransposon families [83]. 

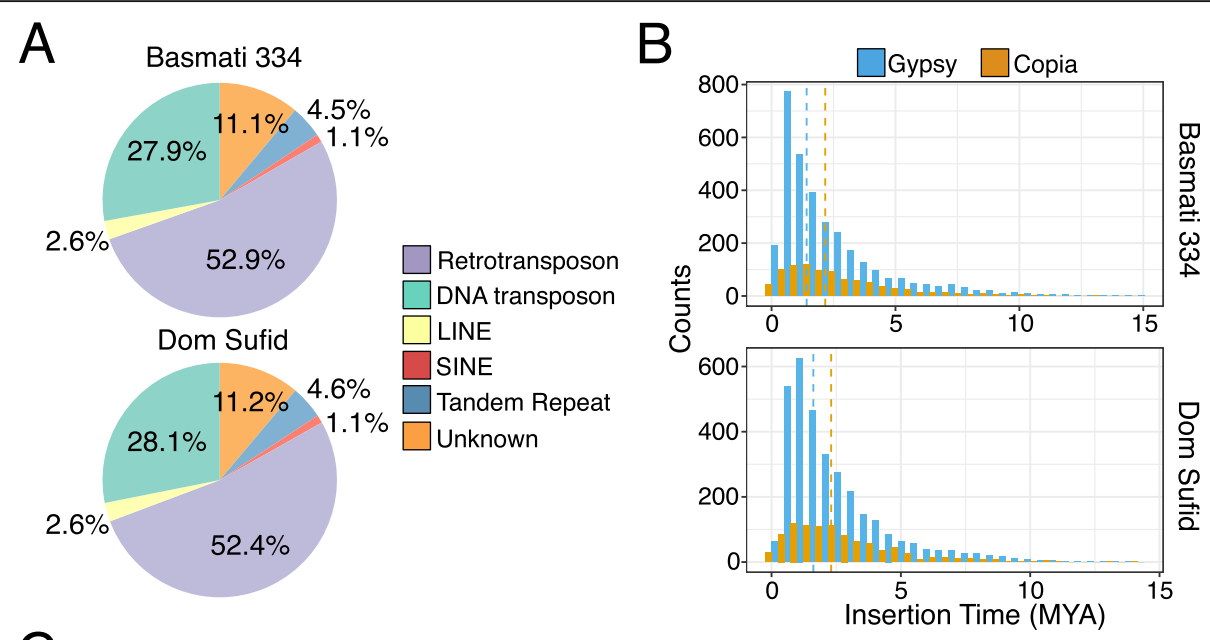

C
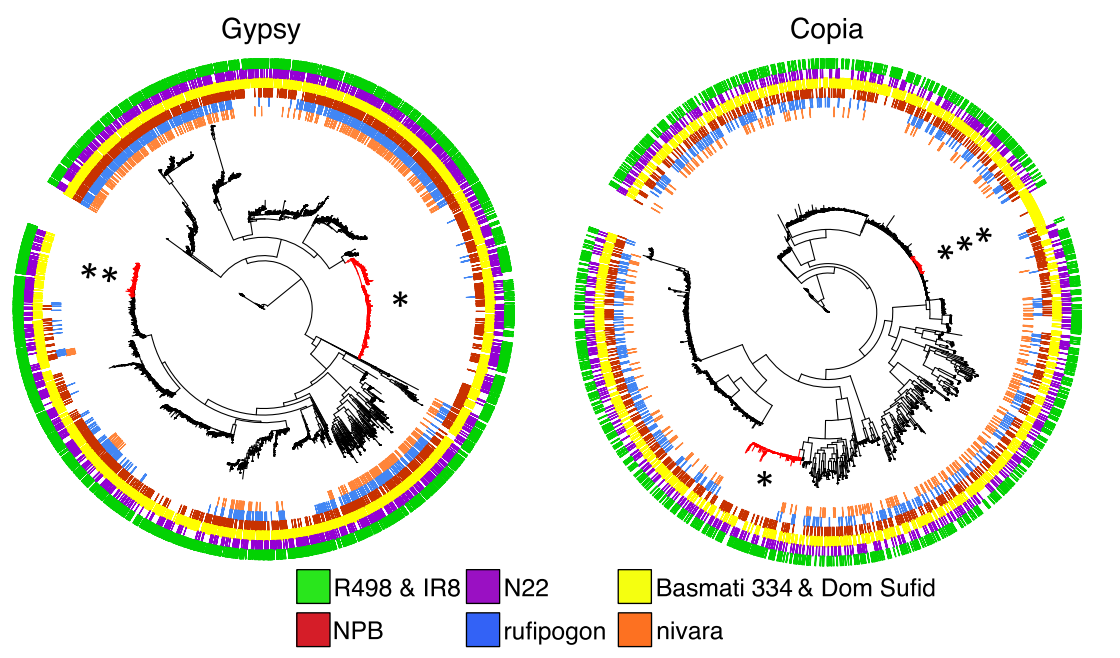

Fig. 4 Repetitive DNA landscape of the Basmati 334 and Dom Sufid genomes. a Proportion of repetitive DNA content in the circum-basmati genomes represented by each repeat family. b Distribution of insert times for the gypsy and copia LTR retrotransposons. $\mathbf{c}$ Phylogeny of gypsy and copia LTR retrotransposons based on the rve gene. LTR retrotransposons were annotated from the reference genomes of domesticated and wild rice

The gypsy and copia superfamilies each contained a clade in which the majority of elements originated within $O$. sativa, and only present among the four domesticated rice variety groups (Fig. 4c, single star; see Additional files 2: Tables S12 and S13 for their genome coordinates). Elements in the gypsy superfamily phylogenetic clade had sequence similarity (963 out of the 1837 retrotransposons) to elements of the hopi family [84], while elements in the copia superfamily phylogenetic clade had sequence similarity ( 88 out of the 264) to elements in the osr4 family [85]. Elements of the hopi family are found in high copy number in genomes of domesticated rice varieties [86] and this amplification has happened recently [87].

Several retrotransposon clades were restricted to certain rice variety groups. The gypsy superfamily harbored a phylogenetic clade whose elements were only present in genomes of circum-aus, circum-basmati, and indica varieties (Fig. 4c, double star; see Additional file 2: Table S14 for their genome coordinates), while we observed a clade comprised mostly of circum-basmati-specific elements within the copia superfamily (Fig. 4c, triple star; see Additional file 2: Table S15 for their genome coordinates). Only a few members of the gypsy-like clade had sequence similarity ( 7 out of 478 ) to elements of the rire3 [88] and rn215 [89] families. Members of both families are known to be present in high copy numbers in genomes of domesticated rice varieties, but their abundance differs between the japonica and indica variety groups [86], suggesting a rire3- or rn215-like element expansion in the circum-aus, circum-basmati, and indica genomes. A majority of the circum-basmati-specific copia-like elements had sequence similarity (109 out of 113) to members of the houba family [84], which are found in high copy numbers in certain individuals, but in lower frequency across the rice population [86]. This 
suggests the houba family might have undergone a recent expansion specifically within the circum-basmati genomes.

\section{Phylogenomic analysis on the origins of circum-basmati rice}

We estimated the phylogenetic relationships within and between variety groups of domesticated Asian rice. Our maximum likelihood phylogenetic tree, based on fourfold degenerate sites from the Nipponbare coding sequences (Fig. 5a), showed that each cultivar was monophyletic with respect to its variety group of origin. In addition, the circum-basmati group was sister to japonica rice, while the circum-aus group was sister to indica. Consistent with previous observations, the wild rice $O$. nivara and O. rufipogon were sister to the circum-aus and japonica rice, respectively [14]. While this suggests that each domesticated rice variety group may have had independent wild progenitors of origin, it should be noted that recent hybridization between wild and domesticated rice $[90,91]$ could lead to similar phylogenetic relationships.

To further investigate phylogenetic relationships between circum-basmati and japonica, we examined phylogenetic topologies of each gene involving the trio Basmati 334, Nipponbare, and O. rufipogon. For each gene, we tested which of three possible topologies for a rooted three-species tree-i.e., [(P1, P2), P3], O, where $\mathrm{O}$ is outgroup $\mathrm{O}$. barthii and $\mathrm{P} 1, \mathrm{P} 2$, and $\mathrm{P} 3$ are Basmati 334 (or Dom Sufid), Nipponbare, and O. rufipogon, respectively-were found in the highest proportion. For the trio involving Basmati 334, Nipponbare, and O. rufipogon, there were 7581 genes (or 32.6\%), and for the trio involving Dom Sufid, Nipponbare, and O. rufipogon, there were 7690 genes (or 33.1\%), which significantly rejected one topology over the other two using an Approximately Unbiased (AU) topology test [92]. In both trios, the majority of those genes supported a topology that grouped circum-basmati and Nipponbare as sister to each other (Fig. 5b; 3881 [or 51.2\%] and 4407 [or 57.3\%] genes for Basmati 334 and Dom Sufid, respectively). A lower number of genes (3018 [or 39.8\%] and 2508 [or 32.6\%] genes for Basmati 334 and Dom Sufid, respectively) supported the topology that placed Nipponbare and $O$. rufipogon together.

Our initial topology test suggested that the trio involving Dom Sufid, Nipponbare, and O. rufipogon had a higher proportion of genes supporting the [(circum-basmati, japonica), O. rufipogon] topology compared to the trio involving Basmati 334, Nipponbare, and O. rufipogon (Fig. 5b). This suggested within-population variation in the amount of japonica or $O$. rufipogon ancestry across the circum-basmati genomes due to differences in gene flow. To test for introgression, we employed D-statistics from the ABBA-BABA test $[93,94]$. We conducted ABBA-BABA tests involving the topology [(Basmati 334, Dom Sufid), Nipponbare or $O$. rufipogon] to examine the differences in introgression between the circum-basmati and japonica or O. rufipogon genomes. The results showed significantly positive Dstatistics for the topology [(Basmati 334, Dom Sufid), Nipponbare] (Fig. 5c left panel; $z$-score $=8.42$ and $D=0.27 \pm$ 0.032), indicating that Dom Sufid shared more alleles with japonica than Basmati 334 did due to a history of more admixture with japonica. The D-statistics involving the topology [(Basmati 334, Dom Sufid), O. rufipogon] were also significantly positive (Fig. $5 \mathrm{c}$ left panel; $z$-score $=5.57$ and $D=0.21 \pm 0.038$ ).

\section{Signatures of admixture between circum-basmati and circum-aus rice genomes}

Due to extensive admixture between rice variety group genomes [14], we examined whether the basmati genome was also influenced by gene flow with other divergent rice variety groups (i.e., circum-aus or indica rice). A topology test was conducted for a rooted, three-population species tree. For the trio involving Basmati 334, circum-aus variety N22, and indica variety R498, there were 7859 genes (or 35.3\%), and for the trio involving Dom Sufid, N22, and R498, there were 8109 genes (or 37.8\%), which significantly rejected one topology over the other two after an AU test. In both trios, more than half of the genes supported the topology grouping circum-aus and indica as sisters (Fig. 5d). In addition, more genes supported the topology grouping circum-aus and circum-basmati as sisters than the topology grouping indica and circumbasmati as sisters. This suggested that the circum-aus variety group might have contributed a larger proportion of genes to circum-basmati through gene flow than the indica variety group did.

To test for evidence of admixture, we conducted ABBABABA tests involving trios of the circum-basmati, N22, and R498 genomes. Results showed significant evidence of gene flow between circum-aus and both circum-basmati genomes-Fig. 5c, right panel; $z$-score $=5.70$ and $D=$ $0.082 \pm 0.014$ for topology [(R498, N22), Basmati 334]; and $z$-score $=8.44$ and $D=0.11 \pm 0.013$ for topology [(R498, N22), Dom Sufid]. To test whether there was variability in the circum-aus or indica ancestry in each of the circumbasmati genomes, we conducted ABBA-BABA tests for the topology [(Basmati 334, Dom Sufid), N22 or R498]. Neither of the ABBA-BABA tests involving the topology [(Basmati 334, Dom Sufid), N22] (Fig. 5c, right panel; $z$ score $=1.20$ and $D=0.025 \pm 0.021)$ or the topology [(Basmati 334, Dom Sufid), R498] (Fig. 5c, right panel; $z$-score = -2.24 and $D=-0.06 \pm 0.026$ ) was significant, suggesting the amount of admixture from circum-aus to each of the two circum-basmati genomes was similar.

Because of the significant amount of admixture occurring between the circum-aus and circum-basmatigenomes, we 


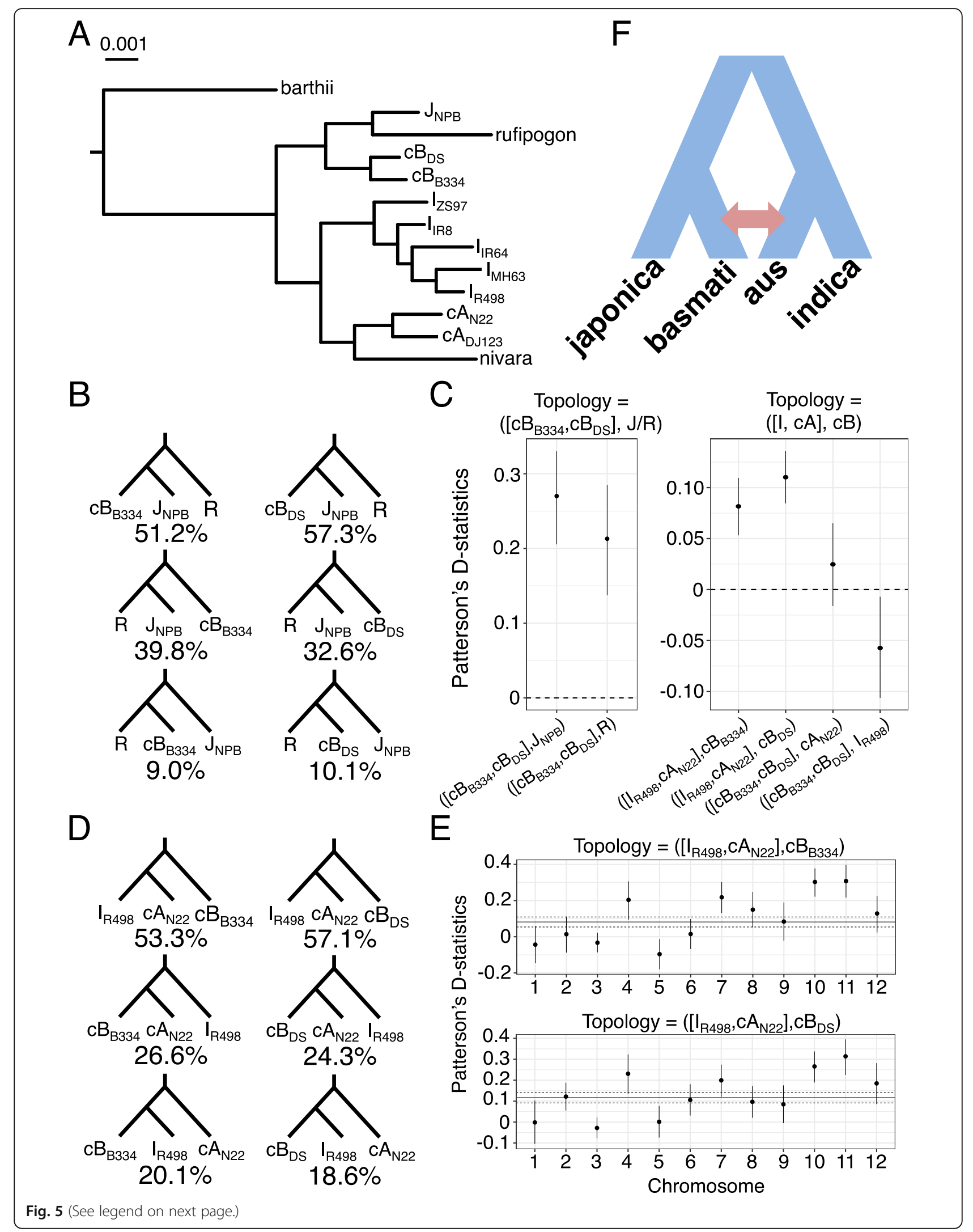


(See figure on previous page.)

Fig. 5 Comparative genomic analysis of circum-basmati rice evolution. The Oryza sativa variety groups are labeled as circum-aus (cA), circum-basmati (CB), indica (I), and japonica $(\mathrm{J})$, and the wild relative is $O$. rufipogon (R). a Maximum likelihood tree based on fourfold degenerate sites. All nodes had over 95\% bootstrap support. b Percentage of genes supporting the topology involving japonica Nipponbare, circum-basmati Basmati 334 (B334) and Dom Sufid (DS), and O. rufipogon after an Approximately Unbiased (AU) test. c Results of ABBA-BABA tests. Shown are median Patterson's D-statistics with $95 \%$ confidence intervals determined from a bootstrapping procedure. For each tested topology, the outgroup was always $O$. barthii. $\mathbf{d}$ Percentage of genes supporting the topology involving circum-aus N22, circum-basmati, and indica R498 after an AU test. e Per-chromosome distribution of D-statistics for the trio involving R498, N22, and each circum-basmati genome. Genome-wide D-statistics with 95\% bootstrap confidence intervals are indicated by the dark and dotted lines. $\mathbf{f}$ Model of admixture events that occurred within domesticated Asian rice. The direction of admixture has been left ambiguous, as the ABBA-BABA test cannot detect the direction of gene flow

examined whether this had affected the topology analysis involving the trio japonica, circum-basmati, and $O$. rufipogon (Fig. 5b). Specifically, we assessed whether the grouping of japonica and $O$. rufipogon as sister species (Fig. 5a) was an evolutionary artifact due to sharing of alleles between circum-basmati and circumaus through admixture. We examined this by conducting the AU test on the four populations involving circum-aus, circum-basmati (Basmati 334 or Dom Sufid), japonica, and O. rufipogon, testing which of the 15 possible topologies for a rooted four-population sample (see Additional file 1: Figure S6 for the 15 topologies tested) was the best fit for each gene. Results showed there were 2774 genes involving Basmati 334 and 2665 genes involving Dom Sufid where the AU test significantly rejected one topology over the other 14 topologies (Additional file 1: Figure S6). The most frequent topology (>30\% of the genes) was one that both grouped japonica and O. rufipogon as sisters and grouped circum-basmati and circum-aus as sisters, which is a topology that occurs when there is admixture occurring between circum-basmati and circum-aus. The second most frequent topology ( $>20 \%$ of the genes) was the species phylogeny (i.e., [(circum-basmati, japonica), $O$. rufipogon]) and this was represented fivefold higher than the remaining 13 topologies. In the end, this result partially explains the discrepancy between the genome-wide tree topology (Fig. 5a) and the gene-specific tree topology (Fig. 5b). The admixture occurring between circumbasmati and circum-aus had led to the spurious genomewide topological relationship.

In sum, the phylogenomic analysis indicated that circumbasmati and japonica share the most recent common ancestor, while circum-aus has admixed with circum-basmati during its evolutionary history (Fig. 5f). We then examined whether admixture from circum-aus had affected each of the circum-basmati chromosomes to a similar degree. For both circum-basmati genomes, most chromosomes had Dstatistics that were not different from the genome-wide Dstatistics value or from zero (Fig. 5e). Exceptions were chromosomes 10 and 11, where the bootstrap D-statistics were significantly higher than the genome-wide estimate.

\section{Population analysis on the origin of circum-basmati rice} Since our analysis was based on single representative genomes from each rice variety group, we compared the results of our phylogenomic analyses to population genomic patterns in an expanded set of rice varieties from different groups. We obtained high coverage $(>14 \times)$ genomic re-sequencing data (generated with Illumina short-read sequencing) from landrace varieties in the $3 \mathrm{~K}$ Rice Genome Project [7] and from circum-basmati rice landraces we re-sequenced. In total, we analyzed 24 circum-aus, 18 circum-basmati, and 37 tropical japonica landraces (see Additional file 2: Table S16 for variety names). The raw Illumina sequencing reads were aligned to the scaffolded Basmati 334 genome and computationally genotyped. A total of 4,594,290 polymorphic sites were called across the three rice variety groups and used for further analysis.

To quantify relationships between circum-aus, circumbasmati, and japonica, we conducted a topology-weighting analysis [95]. For three populations, there are three possible topologies and we conducted localized sliding window analysis to quantify the number of unique subtrees that supported each tree topology. Consistent with the phylogenomic analysis results, the topology weight was the largest for the topology that grouped japonica and circum-basmati as sisters (Fig. 6a; topology weight $=0.481$ with $95 \%$ confidence interval [0.479-0.483]). The topology that grouped circum-aus and circum-basmati together as sisters weighed significantly more (topology weight = 0.318 with $95 \%$ confidence interval [0.316-0.320]) than the topology that grouped japonica and circum-aus as sisters (topology weight $=0.201$ with $95 \%$ confidence interval [0.199-0.203]). This was consistent with the admixture results from the comparative phylogenomic analysis, which detected evidence of gene flow between circum-aus and circum-basmati.

A treemix analysis was conducted for the three domesticated rice population (circum-aus, circum-basmati, and japonica) alongside the wild rice $O$. rufipogon and $O$. barthii (Additional file 1: Figure S7). We fitted zero to three migration edges in the model, and at three migration edges, the model log-likelihood started plateauing 


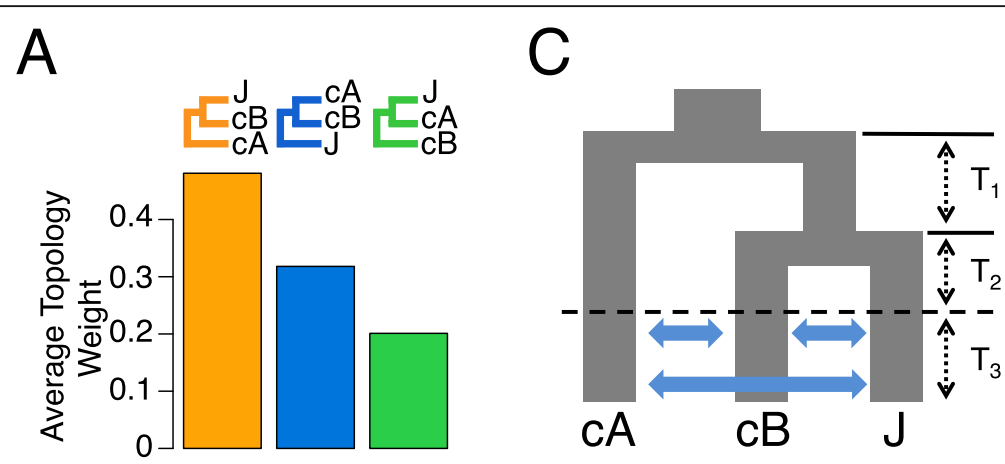

$\mathrm{B}$

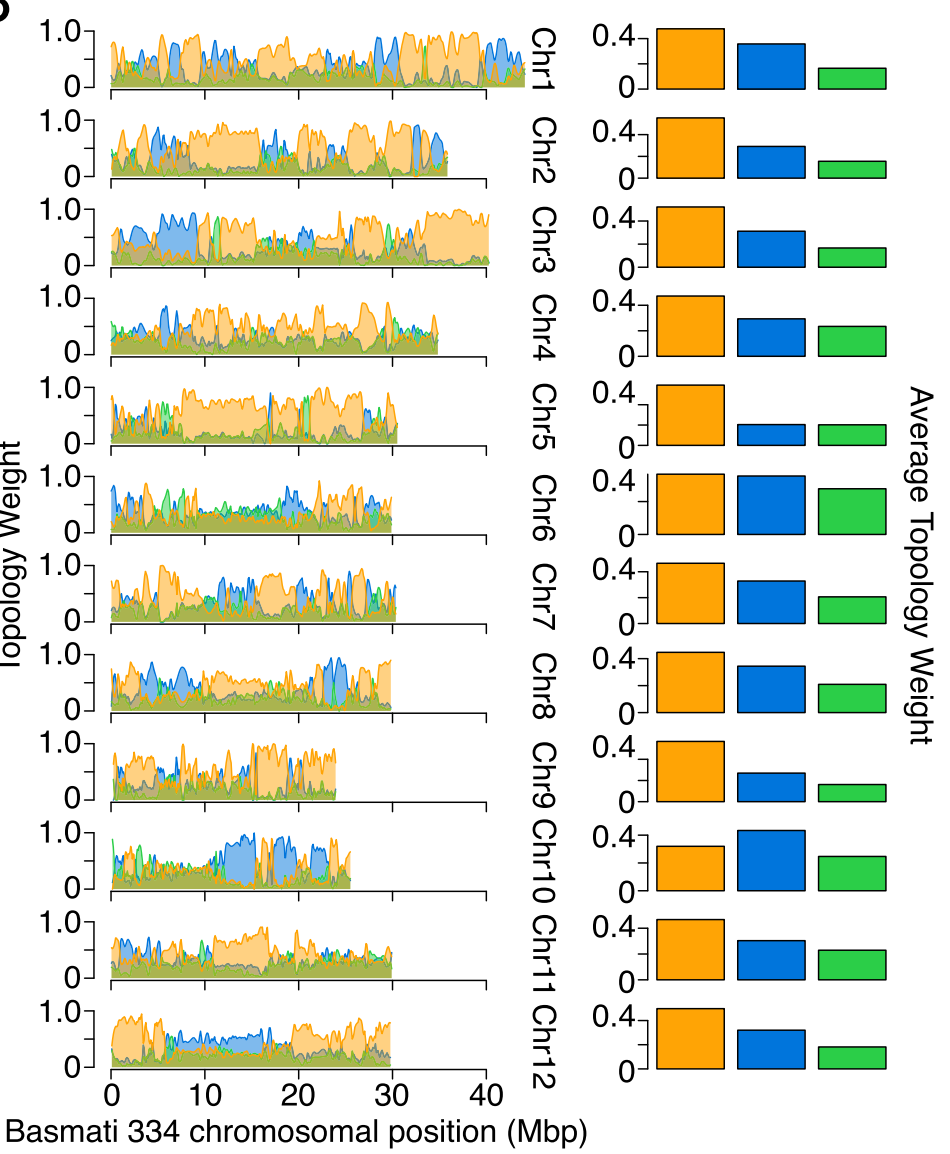

Fig. 6 Population relationships among the circum-aus (CA), circum-basmati (cB), and japonica rice (J). a Sum of genome-wide topology weights for a three-population topology involving trios of the circum-aus, circum-basmati, and japonica rice. Topology weights were estimated across windows with 100 SNPs. b Chromosomal distributions of topology weights involving trios of the circum-aus, circum-basmati, and japonica rice (left), and the sum of the topology weights (right). c Best-fitting $\delta a \delta i$ model for the circum-aus, circum-basmati, and japonica rice. See Additional file 2: Table S17 for parameter estimates

(Additional file 1: Figure S7B). At three migration edges, a migration edge was fitted between circum-aus and circum-basmati (Additional file 1: Figure S7A), consistent with our previous results. In addition, there were migration edges fitted between the wild rice O. rufipogon and circum-basmati and between the wild rice $O$. barthii and japonica. Overall, these migration results were consistent with recent studies that have documented the occurrence of admixture between wild and domesticated rice populations $[74,90,91]$.

We then examined topology weights for each individual chromosome, since the ABBA-BABA tests using the genome assemblies had detected variation in circum-aus ancestry between different chromosomes (Fig. 5e). The results showed that for most of the chromosomes the topology [(japonica, circum-basmati), circum-aus] always 
weighed more than the remaining two topologies. An exception was observed for chromosome 10 where the topology weight grouping circum-aus and circum-basmati as sisters was significantly higher (topology weight $=0.433$ with 95\% confidence interval [0.424-0.442]) than the weight for the genome-wide topology that grouped japonica and circum-basmati as sisters (topology weight $=0.320$ with 95\% confidence interval [0.312-0.328]). This change in predominant topology was still observed when the weights were calculated across wider local windows (Additional file 1: Figure S8). Another exception could be seen for chromosome 6 where the genome-wide topology [(japonica, circum-basmati), circum-aus] (topology weight $=0.367$ with $95 \%$ confidence interval $[0.359-0.374])$ and the admixture topology [(circum-aus, circum-basmati), japonica] (topology weight $=0.355$ with $95 \%$ confidence interval [0.349-0.362]) had almost equal weights. In larger window sizes, the weight of the admixed topology was slightly higher than that of the genomewide topology (Additional file 1: Figure S8).

To estimate the evolutionary/domestication scenario that might explain the observed relationships between the circum-aus, circum-basmati, and japonica groups, we used the diffusion-based approach of the program $\delta a \delta i$ [96] and fitted specific demographic models to the observed allele frequency spectra for the three rice variety groups. Because all three rice groups have evidence of admixture with each other $[7,9,14,16]$, we examined 13 demographic scenarios involving symmetric, asymmetric, and "no migration" models between variety groups, with and without recent population size changes (Additional file 1: Figure S9). To minimize the effect of genetic linkage on the demography estimation, polymorphic sites were randomly pruned in $200-\mathrm{kb}$ windows, resulting in 1918 segregating sites. The best-fitting demographic scenario was one that modeled a period of lineage splitting and isolation, while gene flow only occurred after formation of the three populations and at a later time (Fig. 6c; visualizations of the 2D site frequency spectrum and model fit can be seen in Additional file 1: Figure S10). This best-fitting model was one of the lesser-parameterized models we tested, and the difference in Akaike Information Criterion $(\triangle \mathrm{AIC})$ with the model with the second-highest likelihood was 25.46 (see Additional file 2: Table S17 for parameter estimates and maximum likelihood estimates for each demographic model).

\section{Genetic structure within the circum-basmati group}

We used the circum-basmati population genomic data for the 78 varieties aligned to the scaffolded Basmati 334 genome and called the polymorphic sites segregating within this variety group. After filtering, a total of 4,430, 322 SNPs across the circum-basmati dataset remained, which were used to examine population genetic relationships within circum-basmati.

We conducted principal component analysis (PCA) using the polymorphism data and color-coded each circumbasmati rice variety according to its country of origin (Fig. 7a). The PCA suggested that circum-basmati rice could be divided into three major groups with clear geographic associations: (group 1) a largely Bhutan/Nepal-based group, (group 2) an India/Bangladesh/Myanmar-based group, and (group 3) an Iran/Pakistan-based group. The rice varieties that could not be grouped occupied an ambiguous space across the principal components, suggesting these might represent admixed rice varieties.

To obtain better insight into the ancestry of each rice variety, we used fastSTRUCTURE [97] and varied assumed ancestral population $(K)$ from 2 to 5 groups so the ancestry proportion of each rice variety could be estimated (Fig. 7b). At $K=2$, the India/Bangladesh/ Myanmar and Iran/Pakistan rice groups were shown to have distinct ancestral components, while the Bhutan/ Nepal group was largely an admixture of the other two groups. At $K=3$, the grouping status designated from the PCA was largely concordant with the ancestral components. At $K=4$, most India/Bangladesh/Myanmar rice had a single ancestral component, but Iran/Pakistan rice had two ancestral components that were shared with several Bhutan/Nepal landraces. Furthermore, several of the cultivars from the latter group seemed to form an admixed group with India/Bangladesh/Myanmar varieties. In fact, when a phylogenetic tree was reconstructed using the polymorphic sites, varieties within the India/Bangladesh/ Myanmar and Iran/Pakistan groups formed a monophyletic clade with each other. On the other hand, Bhutan/Nepal varieties formed a paraphyletic group where several clustered with the Iran/Pakistan varieties (Additional file 1: Figure S11).

We then conducted a second fastSTRUCTURE analysis on the circum-basmati population, this time including the japonica and circum-aus populations while varying $K$ from 2 to 5 groups (Additional file 1: Figure S12). From $K=2$ to 5 , the japonica and circum-aus groups always formed two distinct genetic groups. At $K=5$, the three circumbasmati genetic groups that were designated in the first analysis (Fig. 7) were still observed across the circumbasmati population. In lower $K$, we see that the different circum-basmati genetic groups had differing amounts of japonica or circum-aus ancestry. Specifically, the Iran/ Pakistan group had more circum-aus ancestry, while the India/Bangladesh/Myanmar group had more japonica ancestry. The Bhutan/Nepal group again was suggested to be an admixture of the other two circum-basmati rice genetic groups.

In summary, the circum-basmati rice has evolved across a geographic gradient with at least three genetic 

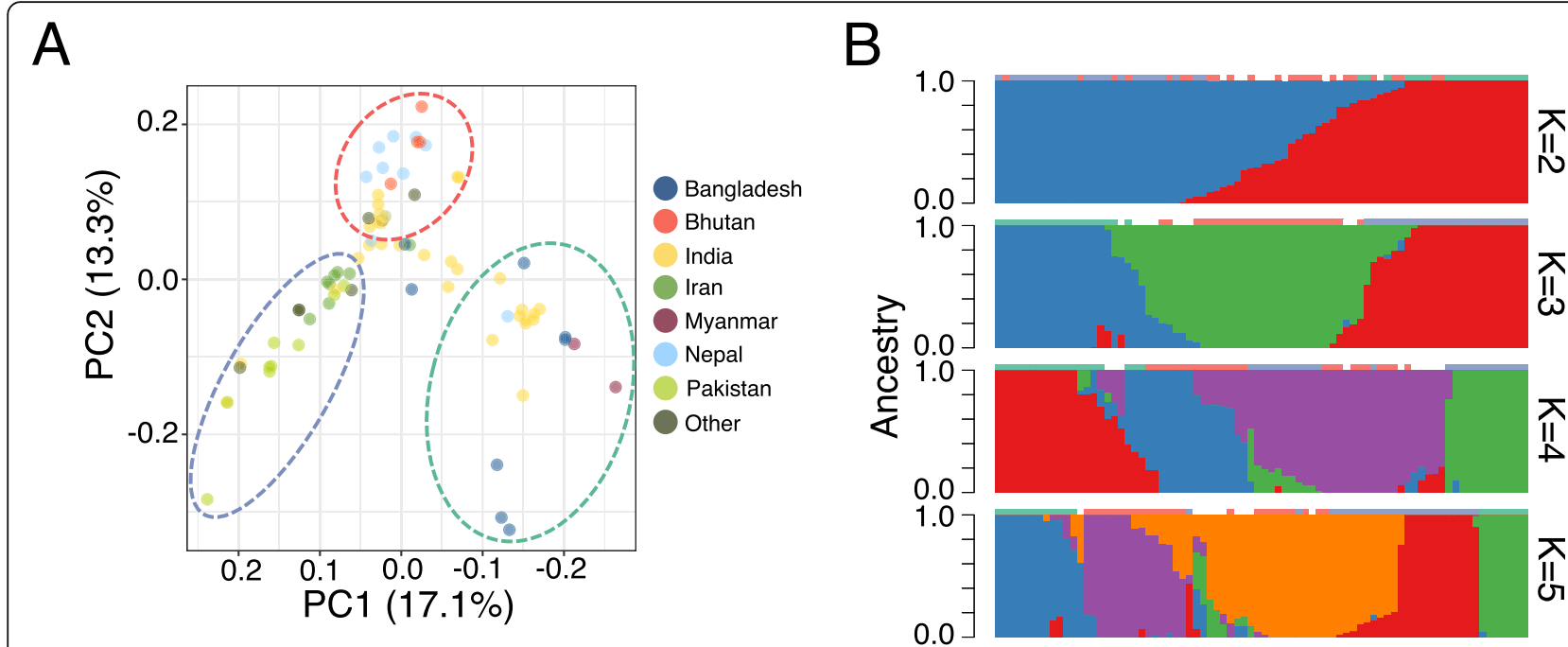

C

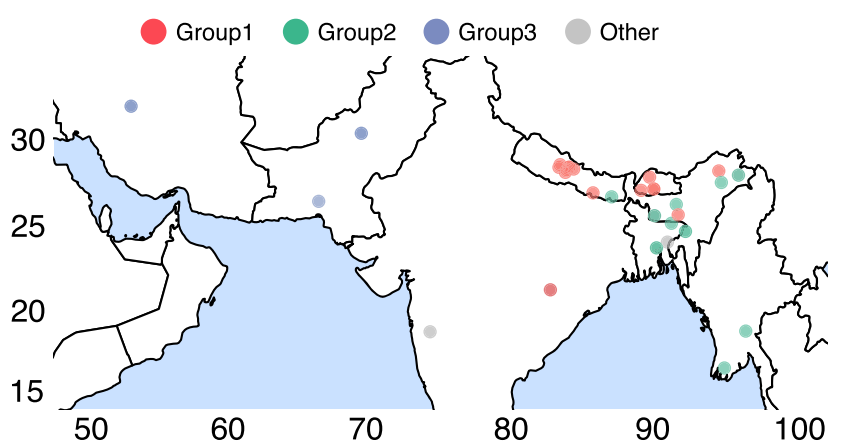

D

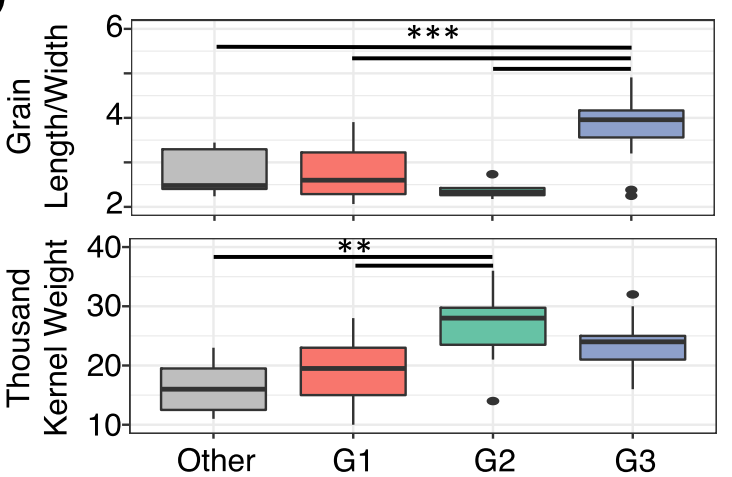

Fig. 7 Population structure within the circum-basmati rice. a PCA plot for the 78-variety circum-basmati rice population genomic dataset. The three genetic groups designated by this study can be seen in the color-coded circles with dashed lines. b Proportion of ancestry plot for $K=2,3,4$, and 5 across the 78 circum-basmati rice varieties. The color-coding from (a) is indicated above each sample's ancestry proportion. c Geographic distribution of the 78 circum-basmati rice varieties with their grouping status color-coded according to a. $\mathbf{d}$ Agronomic measurements for the 78 circum-basmati rice varieties sorted into the three groups designated by this study. Two asterisks indicate $p$ value $<0.01$ and three asterisks indicate $p$ value $<0.001$

groups (Fig. 7c). These existed as distinct ancestral groups that later admixed to form several other circumbasmati varieties. Group 1 and group 3 rice in particular may have experienced greater admixture, while the group 2 landraces remained genetically more isolated from other circum-basmati subpopulations. We also found differences in agronomic traits associated with our designated groups (Fig. 7d). The grain length to width ratio, which is a highly prized trait in certain circum-basmati rice [24], was significantly larger in group 3 Iran/Pakistan varieties. The thousand-kernel weights, on the other hand, were highest for group 2 India/Bangladesh/Myanmar varieties and were significantly higher than those for the ungrouped and group 1 Bhutan/Nepal varieties.

\section{Discussion}

Nanopore sequencing is becoming an increasingly popular approach to sequence and assemble the often large and complex genomes of plants [98-100]. Here, using long-read sequences generated with Oxford Nanopore Technologies' sequencing platform, we assembled genomes of two circum-basmati rice cultivars, with quality metrics that were comparable to other rice variety group reference genome assemblies [37, 40, 41]. With modest genome coverage, we were able to develop reference genome assemblies that represented a significant improvement over a previous circum-basmati reference genome sequence, which had been assembled with a $>3$-fold higher genome coverage than ours, but from short-read sequences [42]. With additional short-read sequencing reads, we were able to correct errors from the nanopore sequencing reads, resulting in two high-quality circumbasmati genome assemblies.

Even with long-read sequence data, developing good plant reference genome sequences still requires additional technologies such as optical mapping or $\mathrm{Hi}-\mathrm{C}$ sequencing for improving assembly contiguity [101-104], 
which can be error prone as well [56]. Our assemblies were also fragmented into multiple contigs, but sizes of these contigs were sufficiently large that we could use reference genome sequences from another rice variety group to anchor the majority of contigs and scaffold them to higherorder chromosome-level assemblies. Hence, with a highly contiguous draft genome assembly, reference genomebased scaffolding can be a cost-efficient and powerful method of generating chromosome-level assemblies.

Repetitive DNA constitutes large proportions of plant genomes [105], and there is an advantage to using longread sequences for genome assembly as it enables better annotation of transposable elements. Many transposable element insertions have evolutionarily deleterious consequences in the rice genome [54, 106, 107], but some insertions could have beneficial effects on the host [108]. Using our genome assembly, we have identified retrotransposon families that have expanded specifically within circumbasmati genomes. While more study will be necessary to understand the functional effects of these insertions, longread sequences have greatly improved the assembly and identification of repeat types.

Due to a lack of archaeobotanical data, the origins of circum-basmati rice have remained elusive. Studies of this variety group's origins have primarily focused on genetic differences that exist between circum-basmati and other Asian rice variety groups [6, 7]. Recently, a study suggested that circum-basmati rice (called "aromatic" in that study) was a product of hybridization between the circum-aus and japonica rice variety groups [17]. This inference was based on observations of phylogenetic relationships across genomic regions that showed evidence of domesticationrelated selective sweeps. These regions mostly grouped circum-basmati with japonica or circum-aus. In addition, chloroplast haplotype analysis indicated that most circumbasmati varieties carried a chloroplast derived from a wild rice most closely related to circum-aus landraces [109]. Our evolutionary analysis of circum-basmati rice genomes generally supported this view. Although our results suggest that circum-basmati had its origins primarily in japonica, we also find significant evidence of gene flow originating from circum-aus, which we detected both in comparative genomic and population genomic analyses. Our results are also consistent with the observation of genome-wide fine-scale admixture tracts that showed japonica rice contributing the highest amount of genetic material to circum-basmati, followed by circum-aus [110]. Demographic modeling indicated a period of isolation among circum-aus, circum-basmati, and japonica, with gene flow occurring only after lineage splitting of each group. Here, our model is consistent with the current view that gene flow is a key evolutionary process associated with the diversification of rice $[10,12-14,16,111,112]$.
Interestingly, we found that chromosome 10 of circum-basmati had an evolutionary history that differed significantly from that of other chromosomes. Specifically, compared to japonica, this chromosome had the highest proportion of presence/absence variation and shared more alleles with circum-aus. Based on this result, we hypothesize that this is largely due to higher levels of introgression from circum-aus into chromosome 10 compared to other chromosomes. Such a deviation of evolutionary patterns on a single chromosome has been observed in the Aquilegia genus [113], but to our knowledge has not been observed elsewhere. Why this occurred is unclear at present, but it may be that selection has driven a higher proportion of circum-aus alleles into chromosome 10 . Future work will be necessary to clarify the consequence of this higher level of admixture on chromosome 10.

Very little is known about population genomic diversity within circum-basmati. Our analysis suggests the existence of at least three genetic groups within this variety group, and these groups showed geographic structuring. Several varieties from group 1 (Bhutan/ Nepal) and group 3 (Iran/Pakistan) had population genomic signatures consistent with an admixed population, while group 2 (India/Bangladesh/Myanmar) was genetically more distinct from the other two subpopulations. In addition, the geographic location of the India/ Bangladesh/Myanmar group largely overlaps the region where circum-aus varieties were historically grown $[114,115]$. Our genome-wide evidence of geographic structuring is also consistent with past studies that examined isozyme loci or simple sequence repeat (SSR) markers in Asian rice, and finding a longitudinal genetic structure from Iran to Myanmar. Isozymes could differentiate the circum-basmati rice from other Asian rice subpopulations (classified as group V in Glaszmann [8]) and the varieties from Iran were especially differentiated at two isozyme loci [116]. SSR markers differentiated the Myanmar group $V$ varieties from the rest of south and west Asian varieties [117]. Based on this study and past evidences, genome-wide survey of an expanded sample of circum-basmati should assist in determining the fine-scale genetic structure of the circum-basmati population.

Given the extensive history of admixture that circumbasmati rice has with circum-aus, the India/Bangladesh/ Myanmar group may have been influenced particularly strongly by gene flow from circum-aus. How these three genetic subpopulations were established may require a deeper sampling with in-depth analysis, but the geographically structured genomic variation shows that the diversity of circum-basmati has clearly been underappreciated. In addition, the Basmati 334 and Dom Sufid varieties, for which we generated genome assemblies in this 
study, both belong to the Iran/Pakistan genetic group. Thus, our study still leaves a gap in our knowledge of genomic variation in the Bhutan/Nepal and India/Bangladesh/ Myanmar genetic groups, and varieties in these groups would be obvious next targets for generating additional genome assemblies.

\section{Conclusions}

In conclusion, our study shows that generating high-quality plant genome assemblies is feasible with relatively modest amounts of resources and data. Using nanopore sequencing, we were able to produce contiguous, chromosomelevel genome assemblies for cultivars in a rice variety group that contains economically and culturally important varieties. Our reference genome sequences have the potential to be important genomic resources for identifying singlenucleotide polymorphisms and larger structural variations that are unique to circum-basmati rice. Analyzing de novo genome assemblies for a larger sample of Asian rice will be important for uncovering and studying hidden population genomic variation too complex to study with only shortread sequencing technology.

\section{Materials and methods}

\section{Plant material}

Basmati 334 (IRGC 27819; GeneSys passport: https://purl. org/germplasm/id/23601903-f8c3-4642-a7fc-516a5bc154

f7) is a basmati (sensu stricto) landrace from Pakistan and was originally donated to the International Rice Research Institute (IRRI) by the Agricultural Research Council (ARC) in Karachi (donor accession ID: PAK. SR. NO. 39). Dom Sufid (IRGC 117265; GeneSys passport: https://purl. org/germplasm/id/fb861458-09de-46c4-b9ca-f5c43982291 9) is a sadri landrace from Iran. Seeds from accessions IRGC 27819 and IRGC 117265 were obtained from the IRRI seed bank, surface-sterilized with bleach, and germinated in the dark on a wet paper towel for 4 days. Seedlings were transplanted individually in pots containing continuously wet soil in a greenhouse at New York University's Center for Genomics and Systems Biology and cultivated under a 12-h day-12-h night photoperiod at $30^{\circ} \mathrm{C}$. Plants were kept in the dark in a growth cabinet under the same climatic conditions for 4 days prior to tissue harvesting. Continuous darkness induced chloroplast degradation, which diminishes the amount of chloroplast DNA that would otherwise end up in the DNA extracted from the leaves.

\section{DNA extractions}

Thirty-six 100-mg samples (3.6 g total) of leaf tissue from a total of 10 one-month-old plants were flashfrozen at harvest for each accession and stored at $80^{\circ} \mathrm{C}$. DNA extractions were performed by isolating the cell nuclei and gently lysing the nuclei to extract intact
DNA molecules [118]. Yields ranged between 140 and $150 \mathrm{ng} / \mu \mathrm{l}$.

\section{Library preparation and nanopore sequencing}

Genomic DNA was visualized on an agarose gel to determine shearing. DNA was size-selected using BluePippin BLF7510 cassette (Sage Science) and high-pass mode ( $>20$ $\mathrm{kb}$ ) and prepared using Oxford Nanopore Technologies' standard ligation sequencing kit SQK-LSK109. FLOMIN106 (R9.4) flowcells were used for sequencing on the GridION X5 platform.

\section{Library preparation and Illumina sequencing}

Extracted genomic DNA was prepared for short-read sequencing using the Illumina Nextera DNA Library Preparation Kit. Sequencing was done on the Illumina HiSeq $2500-$ HighOutput Mode v3 with $2 \times 100$ bp read configuration, at the New York University Genomics Core Facility.

\section{Genome assembly, polishing, and scaffolding}

After completion of sequencing, the raw signal intensity data was used for base calling using flip flop (version 2.3.5) from Oxford Nanopore Technologies. Reads with a mean qscore (quality) greater than 8 and a read length greater than $8 \mathrm{~kb}$ were used and trimmed for adaptor sequences using Porechop (https://github.com/rrwick/ Porechop). Raw nanopore sequencing reads were corrected using the program Саnu [119] and then assembled with the genome assembler Flye [120].

The initial draft assemblies were polished for three rounds using the raw nanopore reads with Racon ver. 1.2.1 [121] and one round with Medaka (https://github.com/ nanoporetech/medaka) from Oxford Nanopore Technologies. Afterwards, reads from Illumina sequencing were used by bwa-mem [122] to align to the draft genome assemblies. The alignment files were then used by Pilon ver. 1.22 [123] for three rounds of polishing.

Contigs were scaffolded using a reference genomeguided scaffolding approach implemented in $\mathrm{RaGOO}$ [56]. Using the Nipponbare genome as a reference, we aligned the circum-basmati genomes using Minimap2 [124]. RaGOO was then used to order the assembly contigs. Space between contigs was artificially filled in with 100 "N" blocks.

Genome assembly statistics were calculated using the bbmap stats.sh script from the BBTools suite (https://jgi. doe.gov/data-and-tools/bbtools/). Completeness of the genome assemblies was evaluated using BUSCO ver. 2.0 [125]. Synteny between the circum-basmati genomes and the Nipponbare genome was visualized using $D-G E$ NIES [126]. Genome-wide dotplot from D-GENIES indicated the initial genome assembly of Dom Sufid had an evidence of a large chromosomal fusion between the 
ends of chromosome 4 and 10. Closer examination of this contig (named contig_28 of Dom Sufid) showed the break point overlapped the telomeric repeat sequence, indicating there had been a misassembly between the ends of chromosome 4 and 10. Hence, contig_28 was broken up into two so that each contig represented the respective chromosome of origin and was then subsequently scaffolded using RaGOO.

Inversions that were observed in the dot plot were computationally verified independently using raw nanopore reads. The long-read-aware aligner ngmlr [55] was used to align the nanopore reads to the Nipponbare genome, after which the long-read-aware structural variation caller sniffles [55] was used to call and detect inversions.

The number of sites aligning to the Nipponbare genome was determined using the Mummer4 package [127]. Alignment delta files were analyzed with the dnadiff suite from the Mummer4 package to calculate the number of aligned sites and the number of differences between the Nipponbare genome and the circum-basmati genomes.

\section{Gene annotation and analysis}

Gene annotation was conducted using the MAKER program [52, 53]. An in-depth description of running $M A K E R$ can be found on the website: https://gist.github.com/daren card/bb1001ac1532dd4225b030cf0cd61ce2. We used published Oryza genic sequences as evidence for the gene modeling process. We downloaded the Nipponbare cDNA sequences from RAP-DB (https://rapdb.dna.affrc.go.jp/) to supply as EST evidence, while the protein sequences from the 13 Oryza species project [37] were used as protein evidence for the MAKER pipeline. Repetitive regions identified from the repeat analysis were used to mask out the repeat regions for this analysis. After a first round of running $M A K E R$, the predicted genes were used by SNAP [128] and Augustus [129] to create a training dataset of gene models, which was then used for a second round of MAKER gene annotation. Orthology between the genes from different rice genomes was determined with Orthofinder ver. 1.1.9 [59]. Ortholog statuses were visualized with the UpSetR package [130].

Gene ontology for the orthogroups that are missing specifically in the circum-basmati was examined by using the japonica Nipponbare gene and conducting a gene ontology enrichment analysis on agriGO v2.0 [131]. Gene ontology enrichment analysis for the circum-basmati-specific orthogroups was conducted first by predicting the function and gene ontology of each circum-basmati genome gene model using the eggnog pipeline [132]. We required an ontology to have more than 10 genes as a member for further consideration, and enrichment was tested through a hypergeometric test using the GOstat package [133].

\section{Repetitive DNA annotation}

The repeat content of each genome assembly was determined using Repeatmasker ver. 4.0.5 (http://www. repeatmasker.org/RMDownload.html). We used the Oryza-specific repeat sequences that were identified from Choi et al. [14] (DOI: https://doi.org/10.5061/ dryad.7cr0q), who had used Repeatmodeler ver. 1.0.8 (http://www.repeatmasker.org/RepeatModeler.html) to de novo-annotate repetitive elements across wild and domesticated Oryza genomes [37].

LTR retrotransposons were annotated using the program LTRharvest [134] with parameters adapted from [135]. LTR retrotransposons were classified into superfamilies [82] using the program RepeatClassifier from the RepeatModeler suite. Annotated LTR retrotransposons were further classified into specific families using the 242 consensus sequences of LTR-RTs from the RetrOryza database [89]. We used blastn [136] to search the RetrOryza sequences, and each of our candidate LTR retrotransposons was identified using the "80-80-80" rule [82]: two TEs belong to the same family if they were $80 \%$ identical over at least $80 \mathrm{bp}$ and $80 \%$ of their length.

Insertion times for the LTR retrotransposons were estimated using the DNA divergence between pairs of LTR sequences [81]. The L-INS-I algorithm in the alignment program $M A F F T$ ver. $7.154 \mathrm{~b}$ [137] was used to align the LTR sequences. PAML ver. 4.8 [138] was used to estimate the DNA divergence between the LTR sequences with the Kimura-2-parameter base substitution model [139]. DNA divergence was converted to divergence time (i.e., time since the insertion of a LTR retrotransposon) approximating a base substitution rate of $1.3 \times 10^{-8}[140]$, which is two times higher than the synonymous site substitution rate.

\section{Presence/absence variation detection}

PAVs between the Nipponbare genome and the circumbasmati assemblies were detected using the Assemblytics suites [60]. Initially, the Nipponbare genome was used as the reference to align the circum-basmati assemblies using the program Minimap2. The resulting SAM files were converted to files in delta format using the sam2delta.py script from the RaGOO suite. The delta files were then uploaded onto the online Assemblytics analysis pipeline (http://assemblytics.com/). Repetitive regions would cause multiple regions in the Nipponbare or circum-basmati genomes to align to one another, and in that case, Assemblytics would call the same region as a PAV multiple times. Hence, any PAV regions that overlapped for at least $70 \%$ of their genomic coordinates were collapsed to a single region.

The combination of ngmlr and sniffles was also used to detect the PAVs that differed between the Nipponbare genome and the raw nanopore reads for the circum- 
basmati rice. Because Assemblytics only detects PAVs in the range of 50 to $100,000 \mathrm{bp}$, we used this window as a size limit to filter out the PAVs called by sniffles. Only PAVs supported by more than 5 reads by sniffles were analyzed.

Assemblytics and sniffles call the breakpoints of PAVs differently. Assemblytics calls a single-best breakpoint based on the genome alignment, while sniffles calls a breakpoint across a predicted interval. To find overlapping PAVs between Assemblytics and sniffles, we added $500 \mathrm{bp}$ upstream and downstream of the Assemblyticspredicted breakpoint positions.

\section{Detecting gene deletions across the circum-basmati population}

Genome-wide deletion frequencies of each gene were estimated using the 78-variety circum-basmati population genomic dataset. For each of the 78 varieties, raw sequencing reads were aligned to the circum-basmati and Nipponbare genomes using bwa-mem. Genome coverage per site was calculated using bedtools genomecov [141]. For each variety, the average read coverage was calculated for each gene, and a gene was designated as deleted if its average coverage was less than $0.05 \times$.

\section{Whole-genome alignment of Oryza genomes assembled de novo}

Several genomes from published studies that were assembled de novo were analyzed. These include domesticated Asian rice genomes from the japonica variety group cv. Nipponbare [33]; the indica variety group cvs. 93-11 [32], IR8 [37], IR64 [38], MH63 [40], R498 [41], and ZS97 [40]; the circum-aus variety group cvs. DJ123 [38], Kasalath [39], and N22 [37]; and the circum-basmati variety group cv. GP295-1 [42]. Three genomes from wild rice species were also analyzed; these were $O$. barthii [35], O. nivara [37], and O. rufipogon [37].

Alignment of the genomes assembled de novo was conducted using the approach outlined in Haudry et al. [142], and this alignment approach has been used in another rice comparative genomic study [14]. Briefly, this involved using the Nipponbare genome as the reference for aligning all other genome assemblies. Alignment between japonica and a query genome was conducted using LASTZ ver. 1.03.73 [143], and the alignment blocks were chained together using the UCSC Kent utilities [144]. For japonica genomic regions with multiple chains, the chain with the highest alignment score was chosen as the single-most orthologous region. This analyzes only one of the multiple regions that are potentially paralogous between the japonica and query genomes, but this was not expected to affect the downstream phylogenomic analysis of determining the origin and evolution of the circum-basmati rice variety group. All pairwise genome alignments between the japonica and query genomes were combined into a multi-genome alignment using MULTIZ [145].

\section{Phylogenomic analysis}

The multi-genome alignment was used to reconstruct the phylogenetic relationships between the domesticated and wild rice. Fourfold degenerate sites based on the gene model of the reference japonica genome were extracted using the msa_view program from the phast package ver. 1.4 [146]. The fourfold degenerate sites were used by $R A x M L$ ver. 8.2.5 [147] to build a maximum likelihoodbased tree, using a general time-reversible DNA substitution model with gamma-distributed rate variation.

To investigate the genome-wide landscape of introgression and incomplete lineage sorting, we examined the phylogenetic topologies of each gene [148]. For a three-species phylogeny using $O$. barthii as an outgroup, there are three possible topologies. For each gene, topology-testing methods [149] can be used to determine which topology significantly fits the gene of interest [14]. RAxML-estimated site-likelihood values were calculated for each gene and the significant topology was determined using the Approximately Unbiased (AU) test [92] from the program CONSEL v. 0.20 [150]. Genes with AU test results with a likelihood difference of 0 were omitted, and the topology with an AU test support of greater than 0.95 was selected.

\section{Testing for evidence of admixture}

Evidence of admixture between variety groups was detected using the ABBA-BABA test D-statistics [93, 94]. In a rooted three-taxon phylogeny [i.e., "((P1,P2),P3),O" where $\mathrm{P} 1, \mathrm{P} 2$, and $\mathrm{P} 3$ are the variety groups of interest and $\mathrm{O}$ is outgroup $O$. barthii], admixture can be inferred from the combination of ancestral ("A") and derived ("B") allelic states of each individual. The ABBA conformation arises when variety groups P2 and P3 share derived alleles, while the BABA conformation is found when P1 and P3 share derived alleles. The difference in the frequency of the ABBA and BABA conformations is measured by the D-statistics, where significantly positive D-statistics indicate admixture between the P2 and P3 variety groups, and significantly negative $\mathrm{D}$-statistics indicate admixture between the P1 and P3 variety groups. The genome was divided into 100,000-bp bins for jackknife resampling and calculation of the standard errors. The significance of the D-statistics was calculated using the $Z$-test, and $\mathrm{D}$-statistics with $z$-scores greater than $|3.9|(p<0.0001)$ were considered significant.

\section{Population genomic analysis}

We downloaded FASTQ files from the 3K Rice Genome Project [7] for rice varieties that were determined to be 
circum-basmati varieties in that project. An additional 8 circum-basmati varieties were sequenced on the Illumina sequencing platform as part of this study. The raw reads were aligned to the scaffolded Basmati 334 genome using the program bwa-mem. PCR duplicates were determined computationally and removed using the program picard version 2.9.0 (http://broadinstitute.github.io/pic ard/). Genotype calls for each site were conducted using the GATK HaplotypeCaller engine using the option "-ERC GVCF." The output files were in the genomic variant call format (gVCF), and the gVCFs from each variety were merged using the GATK GenotypeGVCFs engine.

SNP and INDEL variants from the population variant file were filtered independently using the GATK bestpractice hard filter pipeline [151]. SNP variants within 5 bps of an INDEL variant were filtered. Vcftools version 0.1.15 [152] was used to filter sites for which genotypes were not called for more than $20 \%$ of the varieties. Because domesticated rice is an inbreeding species, we also implemented a heterozygosity filter by filtering out sites that had a heterozygote genotype in more than $5 \%$ of the samples using the program vcffilterjdk.jar from the jvarkit suite (https://figshare.com/articles/JVarkit_java_ based_utilities_for_Bioinformatics/1425030). Missing genotypes were imputed and phased using Beagle version 4.1 [153].

To examine the within-circum-basmati variety group population structure, we first randomly pruned the sites by sampling a polymorphic site every $200,000 \mathrm{bp}$ using plink [154]. Plink was also used to conduct a principal component analysis. Ancestry proportions of each sample were estimated using fastSTRUCTURE [97]. A neighborjoining tree was built by calculating the pairwise genetic distances between samples using the Kronecker delta function-based equation [155]. From the genetic distance matrix, a neighbor-joining tree was built using the program FastME [156].

\section{Evolutionary relationships among the circum-basmati, circum-aus, and japonica populations}

To investigate the evolutionary origins of the circumbasmati population, we focused on the landrace varieties that had been sequenced with a genome-wide coverage of greater than $14 \times$. The population data for the circumaus and japonica populations were obtained from the $3 \mathrm{~K}$ Rice Genome Project [7], from which we also analyzed only the landrace varieties that had been sequenced with a genome-wide coverage greater than $14 \times$. For an outgroup, we obtained $O$. barthii sequencing data from previous studies $[35,74]$ and focused on the samples that were not likely to be feralized rice [74]. The Illumina reads were aligned to the scaffolded Basmati 334 genome and SNPs were called and filtered according to the procedure outlined in the "Population genomic analysis" section.

We examined the genome-wide local topological relationship using twisst [95]. Initially, a sliding window analysis was conducted to estimate the local phylogenetic trees in windows with a size of 100 or 500 polymorphic sites using $R A x M L$ with the GTRCAT substitution model. The script raxml_sliding_windows.py from the genomics_general package by Simon Martin (https://github. com/simonhmartin/genomics_general/tree/master/phylo) was used. The "complete" option of twisst was used to calculate the exact weighting of each local window.

\section{Treemix analysis}

A past study by Wang et al. [90] had found evidence of admixture between domesticated rice and wild rice in a dataset of genome sequences from a global sample of rice [16]. Therefore, we obtained O. rufipogon genome data from a separate study that sequenced five samples to a high coverage $(>10 \times)$ [157]. The $O$. rufipogon population sample was combined with the population genomic dataset from the section "Evolu tionary relationships among the circum-basmati, cir cum-aus, and japonica populations." Polymorphic sites were randomly selected every $200 \mathrm{kbp}$, and this dataset was used by Treemix version 1.13 [158] to fit migration edges on a bifurcating tree.

\section{$\delta a \delta i$ demographic model}

The demography model underlying the evolution of circum-basmati rice was tested using the diffusion approximation method of $\delta a \delta i$ [96]. A visual representation of the 13 demographic models that were examined can be seen in Additional file 1: Figure S7. The population group and genotype calls used in the twisst analysis were also used to calculate the site allele frequencies. To conduct a $\delta a \delta i$ analysis for three populations with polarized allele frequency spectrum, the polymorphic sites were polarized using the $O$. barthii reference genome. Using the Basmati 334 reference genome, the O. barthii genome was aligned using the same procedure outlined in the section "Whole-genome alignment of Oryza genomes assembled de novo." This genome alignment was then used to determine the outgroup sequence status for every polymorphic site.

We optimized the model parameter estimates using the Nelder-Mead method and randomly perturbed the parameter values for four rounds. Parameter values were perturbed for threefold, twofold, twofold, and onefold in each subsequent round, while the perturbation was conducted for 10, 20, 30, and 40 replicates in each subsequent round. In each round, parameter values from the best likelihood model of the previous round were used as the starting parameter values for the next round. 
Parameter values from the round with the highest likelihood were chosen to parameterize each demographic model. Akaike Information Criteria (AIC) values were used to compare demography models. The demography model with the lowest AIC was chosen as the bestfitting model.

\section{Agronomic trait measurements}

Data on geolocation of collection as well as on seed dimensions and seed weight for each of the circumbasmati landrace varieties included in this study were obtained from passport data included in the online platform Genesys (https://www.genesys-pgr.org/welcome).

\section{Supplementary information}

Supplementary information accompanies this paper at https://doi.org/10. 1186/s13059-020-1938-2.

Additional file 1: Figure S1. Dot plot comparing chromosome 6 of japonica variety Nipponbare to circum-aus variety N22 and indica variety R498. Figure S2. Distribution of the proportion of missing nucleotides for japonica variety Nipponbare gene models across the orthologous nonjaponica genomic regions. Figure S3. Effect of coverage threshold to call a deletion and the total number of deletion calls for samples with various genome coverage. Figure S4. Density of presence-absence variation (PAV) per 500,000 bp window for each chromosome. Figure S5. Insertion time of LTR retrotransposon in various Oryza variety group genomes. Figure S6. Approximately Unbiased (AU) test result for a 4 population. Figure S7. Treemix result for japonica, circum-basmati, circum-aus, 0. rufipogon, and outgroup 0 . barthii. Figure S8. Genome-wide topology weight from 500 SNP size window. Figure S9. 13 demographic models tested by a i. Figure S10. a i model fit for the best-fitting demographic model. Figure S11. Neighbor-joining phylogenetic tree of the 78 circumbasmati population sample. Figure S12. Proportion of ancestry plot for $\mathrm{K}=2$ to 5 across the 78 circum-basmati rice varieties, and the japonica and circum-aus population studied in Fig. 6c.

Additional file 2: Table S1. Inversion detect by sniffles in the Nipponbare reference genome. Table $\mathbf{S 2}$. The 78 circum-basmati samples with Illumina sequencing result used in this study. Table S3. Names of the Basmati 334 and Dom Sufid genome gene models that had a deletion frequency of zero across the population. Table S4. Names of the Basmati 334 and Dom Sufid genome gene models that had a deletion frequency of above 0.3 and omitted from down stream analysis. Table S5. Orthogroup status for the Basmati 334, Dom Sufid, R498, Nipponbare, and N22 genome gene models. Table S6. Count and repeat types of the presence-absence variation (PAV) in the Basmati 334 or Dom Sufid genome in comparison to the Nipponbare genome. Table S7. Gene ontology results for orthogroups where gene members from the circum-basmati are missing. Table S8. Gene ontology results for orthogroups where gene members from circum-aus, indica, and japonica are missing. Table S9. Population frequency across the 78 circum-basmati samples for orthogroups that were specifically missing a gene in the Basmati 334 and Dom Sufid genome gene models. Table S10. Genome coordinates of the LTR retrotransposons of the Basmati 334 genomes. Table S11. Genome coordinates of the LTR retrotransposons of the Dom Sufid genomes. Table S12. Genome coordinates of the Gypsy elements indicated with a single star in Fig. 3. Table S13. Genome coordinates of the Copia elements indicated with a single star in Fig. 3. Table S14. Genome coordinates of the Gypsy elements indicated with a double star in Fig. 3. Table S15. Genome coordinates of the Copia elements indicated with a triple star in Fig. 3. Table S16. The 82 Oryza population samples with Illumina sequencing result used in this study. Table S17. a i parameter estimates for the 13 different demographic models. See Additional file 1: Figure $\$ 9$ for visualization of the estimating parameters. Additional file 3. Review history.

\section{Acknowledgements}

We thank Katherine Dorph for the assistance with growing and maintaining the plants and Adrian Platts for the computational support. We thank Rod Wing, David Kudrna, and Jayson Talag from Arizona Genomics Institute with the high-molecular weight DNA extraction. We thank the New York University Genomics Core Facility for sequencing support and the New York University High Performance Computing for supplying the computational resources.

Peer review information

Kevin Pang was the primary editor of this article and handled its editorial process and peer review in collaboration with the rest of the editorial team.

\section{Review history}

The review history is available as Additional file 3.

\section{Authors' contributions}

JYC, SCG, SZ, and MDP conceived the project and its components. JYC, SCG, and $S Z$ prepared the sample material for sequencing. $X D, P R, E D H$, and $S J$ conducted the genome sequencing and assembling. JYC, ZNL, and SCG performed the data analysis. JYC and ZNL prepared the figures and tables. JYC and MDP wrote the manuscript with help from ZNL and SCG. All authors read and approved the final manuscript.

\section{Funding}

This work was supported by grants from the Gordon and Betty Moore Foundation through Grant GBMF2550.06 to S.C.G. and from the National Science Foundation Plant Genome Research Program (IOS-1546218), the Zegar Family Foundation (A16-0051) and the NYU Abu Dhabi Research Institute (G1205) to M.D.P. The funding body had no role in the design of the study and collection, analysis, and interpretation of data and in writing the manuscript.

\section{Availability of data and materials}

Raw nanopore sequencing FAST5 files generated from this study are available at the European Nucleotide Archive under bioproject ID PRJEB28274 (ERX3327648-ERX3327652) for Basmati 334 [159] and PRJEB32431 (ERX3334790-ERX3334793) for Dom Sufid [160]. Associated FASTQ files are available under ERX3498039-ERX3498043 for Basmati 334 and ERX3498024ERX3498027 for Dom Sufid. Illumina sequencing generated from this study can be found under bioproject ID PRJNA422249 [161] and PRJNA557122 [162]. A genome browser for both genome assemblies can be found at http://purugganan-genomebrowser.bio.nyu.edu/cgi-bin/hgTracks?db=Bas mati334 for Basmati 334 and http://purugganan-genomebrowser.bio.nyu. edu/cgi-bin/hgTracks?db=DomSufid for Dom Sufid. All data including the assembly, annotation, genome alignment, and population VCFs generated from this study can be found at Zenodo (https://doi.org/10.5281/zenodo. 3355330) [163].

Ethics approval and consent to participate Not applicable.

\section{Consent for publication}

Not applicable.

\section{Competing interests}

XD, PR, EDH, and SJ are employees of Oxford Nanopore Technologies and are shareholders and/or share option holders. The other authors declare that they have no competing interests.

\section{Author details}

${ }^{1}$ Center for Genomics and Systems Biology, Department of Biology, New York University, New York, NY, USA. ${ }^{2}$ Oxford Nanopore Technologies, New York, NY, USA. ${ }^{3}$ New York Genome Center, New York, NY, USA. ${ }^{4}$ Center for Genomics and Systems Biology, NYU Abu Dhabi Research Institute, New York University Abu Dhabi, Abu Dhabi, United Arab Emirates. 
Received: 13 August 2019 Accepted: 17 January 2020

Published online: 05 February 2020

\section{References}

1. Gnanamanickam SS. Rice and its importance to human life. In: Biological control of rice diseases. Dordrecht: Springer Netherlands; 2009. p. 1-11. https://doi.org/10.1007/978-90-481-2465-7_1.

2. Matsuo T, Futsuhara Y, Kikuchi F, Yamaguchi $\mathrm{H}$. Science of the rice plant. Tokyo: Food and Agriculture Policy Research Center; 1997.

3. Gross BL, Zhao Z. Archaeological and genetic insights into the origins of domesticated rice. Proc Natl Acad Sci U S A. 2014:111:6190-7.

4. Nadir S, Khan S, Zhu Q, Henry D, Wei L, Lee DS, et al. An overview on reproductive isolation in Oryza sativa complex. AoB Plants. 2018;10:ply060.

5. Fuller DQ, Sato Y-I, Castillo C, Qin L, Weisskopf AR, Kingwell-Banham EJ, et al. Consilience of genetics and archaeobotany in the entangled history of rice. Archaeol Anthropol Sci. 2010;2:115-31.

6. Garris AJ, Tai TH, Coburn J, Kresovich S, McCouch S. Genetic structure and diversity in Oryza sativa L. Genetics. 2005;169:1631-8.

7. Wang W, Mauleon R, Hu Z, Chebotarov D, Tai S, Wu Z, et al. Genomic variation in 3,010 diverse accessions of Asian cultivated rice. Nature. 2018; 557:43-9.

8. Glaszmann JC. Isozymes and classification of Asian rice varieties. Theoret Appl Genetics. 1987;74:21-30.

9. He Z, Zhai W, Wen H, Tang T, Wang Y, Lu X, et al. Two evolutionary histories in the genome of rice: the roles of domestication genes. PLoS Genet. 2011; 7:e1002100.

10. Fuller DQ. Pathways to Asian civilizations: tracing the origins and spread of rice and rice cultures. Rice. 2012;4:78-92.

11. Meyer RS, Purugganan MD. Evolution of crop species: genetics of domestication and diversification. Nat Rev Genet. 2013;14:840-52.

12. Huang $X$, Han B. Rice domestication occurred through single origin and multiple introgressions. Nat Plants. 2015;2:15207.

13. Castillo CC, Tanaka K, Sato Y-I, Ishikawa R, Bellina B, Higham C, et al. Archaeogenetic study of prehistoric rice remains from Thailand and India: evidence of early japonica in South and Southeast Asia. Archaeol Anthropol Sci. 2016;8:523-43

14. Choi JY, Platts AE, Fuller DQ, Hsing Y-I, Wing RA, Purugganan MD. The rice paradox: multiple origins but single domestication in Asian rice. Mol Biol Evol. 2017:34:969-79.

15. Choi JY, Purugganan MD. Multiple origin but single domestication led to Oryza sativa. G3: genes, genomes. Genetics. 2018;8:797-803.

16. Huang X, Kurata N, Wei X, Wang Z-X, Wang A, Zhao Q, et al. A map of rice genome variation reveals the origin of cultivated rice. Nature. 2012; 490:497-501.

17. Civáň $P, C$ raig $H$, Cox CJ, Brown TA. Three geographically separate domestications of Asian rice. Nat Plants. 2015:1:15164.

18. Wang ZY, Zheng FQ, Shen GZ, Gao JP, Snustad DP, Li MG, et al. The amylose content in rice endosperm is related to the post-transcriptional regulation of the waxy gene. Plant J. 1995;7:613-22.

19. Sweeney MT, Thomson MJ, Pfeil BE, McCouch S. Caught red-handed: Rc encodes a basic helix-loop-helix protein conditioning red pericarp in rice. Plant Cell. 2006:18:283-94.

20. Konishi S, Izawa T, Lin SY, Ebana K, Fukuta Y, Sasaki T, et al. An SNP caused loss of seed shattering during rice domestication. Science. 2006:312:1392-6.

21. Kovach MJ, Calingacion MN, Fitzgerald MA, McCouch SR. The origin and evolution of fragrance in rice (Oryza sativa L.). Proc Natl Acad Sci U S A. 2009;106:14444-9.

22. Xu K, Xu X, Fukao T, Canlas P, Maghirang-Rodriguez R, Heuer S, et al. Sub1A is an ethylene-response-factor-like gene that confers submergence tolerance to rice. Nature. 2006:442:705-8.

23. Bin Rahman ANMR, Zhang J. Preferential geographic distribution pattern of abiotic stress tolerant rice. Rice. 2018;11:10.

24. Singh $R$, Singh $U$, Khush $G$. Aromatic rices. New Delhi: Oxford \& IBH Publishing Co Pvt Ltd; 2000.

25. Bradbury LMT, Gillies SA, Brushett DJ, Waters DLE, Henry RJ. Inactivation of an aminoaldehyde dehydrogenase is responsible for fragrance in rice. Plant Mol Biol. 2008:68:439-49.

26. Chen S, Yang Y, Shi W, Ji Q, He F, Zhang Z, et al. Badh2, encoding betaine aldehyde dehydrogenase, inhibits the biosynthesis of 2-acetyl-1-pyrroline, a major component in rice fragrance. Plant Cell. 2008;20:1850-61.
27. Zhao K, Tung C-W, Eizenga GC, Wright MH, Ali ML, Price AH, et al. Genomewide association mapping reveals a rich genetic architecture of complex traits in Oryza sativa. Nat Commun. 2011;2:467.

28. Heather JM, Chain B. The sequence of sequencers: the history of sequencing DNA. Genomics. 2016;107:1-8.

29. Michael TP, VanBuren R. Progress, challenges and the future of crop genomes. Curr Opin Plant Biol. 2015;24:71-81.

30. Jiao $W-B$, Schneeberger $K$. The impact of third generation genomic technologies on plant genome assembly. Curr Opin Plant Biol. 2017; 36:64-70.

31. Li C, Lin F, An D, Wang W, Huang R. Genome sequencing and assembly by long reads in plants. Genes. 2017;9. https://doi.org/10.3390/genes9010006.

32. Yu J, Hu S, Wang J, Wong GK-S, Li S, Liu B, et al. A draft sequence of the rice genome (Oryza sativa L. ssp. indica). Science. 2002;296:79-92.

33. International Rice Genome Sequencing Project. The map-based sequence of the rice genome. Nature. 2005;436:793-800.

34. Chen J, Huang Q, Gao D, Wang J, Lang Y, Liu T, et al. Whole-genome sequencing of Oryza brachyantha reveals mechanisms underlying Oryza genome evolution. Nat Commun. 2013:4:1595.

35. Wang M, Yu Y, Haberer G, Marri PR, Fan C, Goicoechea JL, et al. The genome sequence of African rice (Oryza glaberrima) and evidence for independent domestication. Nat Genet. 2014;46:982-8.

36. Zhang Y, Zhang S, Liu H, Fu B, Li L, Xie M, et al. Genome and comparative transcriptomics of African wild rice Oryza longistaminata provide insights into molecular mechanism of rhizomatousness and self-incompatibility. Mol Plant. 2015;8:1683-6.

37. Stein JC, Yu Y, Copetti D, Zwickl DJ, Zhang L, Zhang C, et al. Genomes of 13 domesticated and wild rice relatives highlight genetic conservation, turnover and innovation across the genus Oryza. Nat Genet. 2018;50:285

38. Schatz MC, Maron LG, Stein JC, Wences A, Gurtowski J, Biggers E, et al. Whole genome de novo assemblies of three divergent strains of rice, Oryza sativa, document novel gene space of aus and indica. Genome Biol. 2014; 15:506

39. Sakai $H$, Kanamori $H$, Arai-Kichise $Y$, Shibata-Hatta M, Ebana K, Oono $Y$, et al. Construction of pseudomolecule sequences of the aus rice cultivar Kasalath for comparative genomics of Asian cultivated rice. DNA Res. 2014;21:397-405.

40. Zhang J, Chen L-L, Xing F, Kudrna DA, Yao W, Copetti D, et al. Extensive sequence divergence between the reference genomes of two elite indica rice varieties Zhenshan 97 and Minghui 63. Proc Natl Acad Sci U S A. 2016; 113:E5163-71.

41. Du H, Yu Y, Ma Y, Gao Q, Cao Y, Chen Z, et al. Sequencing and de novo assembly of a near complete indica rice genome. Nat Commun. 2017;8: 15324.

42. Zhao Q, Feng Q, Lu H, Li Y, Wang A, Tian Q, et al. Pan-genome analysis highlights the extent of genomic variation in cultivated and wild rice. Nat Genet. 2018;50:278-84

43. Jain S, Jain RK, McCouch SR. Genetic analysis of Indian aromatic and quality rice (Oryza sativa L.) germplasm using panels of fluorescently-labeled microsatellite markers. Theor Appl Genet. 2004;109:965-77.

44. Vikram P, Swamy BPM, Dixit S, Ahmed H, Cruz MTS, Singh AK, et al. Bulk segregant analysis: "An effective approach for mapping consistent-effect drought grain yield QTLs in rice.". Field Crop Res. 2012;134:185-92.

45. McNally KL, Childs KL, Bohnert R, Davidson RM, Zhao K, Ulat VJ, et al. Genomewide SNP variation reveals relationships among landraces and modern varieties of rice. Proc Natl Acad Sci. 2009;106:12273-8.

46. McNally KL, Bruskiewich $\mathrm{R}$, Mackill $\mathrm{D}$, Buell $\mathrm{CR}$, Leach JE, Leung $\mathrm{H}$. Sequencing multiple and diverse rice varieties. Connecting whole-genome variation with phenotypes. Plant Physiol. 2006;141:26-31.

47. Jain M, Olsen HE, Paten B, Akeson M. The Oxford Nanopore MinlON: delivery of nanopore sequencing to the genomics community. Genome Biol. 2016:17:239.

48. Chen S, Huang Z, Zeng L, Yang J, Liu Q, Zhu X. High-resolution mapping and gene prediction of Xanthomonas Oryzae pv. Oryzae resistance gene Xa7. Mol Breed. 2008;22:433-41.

49. Ullah I, Jamil S, Iabal MZ, Shaheen HL, Hasni SM, Jabeen S, et al. Detection of bacterial blight resistance genes in basmati rice landraces. Genet Mol Res. 2012;11:1960-6.

50. Sandhu N, Kumar A, Sandhu N, Kumar A. Bridging the rice yield gaps under drought: QTLs, genes, and their use in breeding programs. Agronomy. 2017;7:27. 
51. Henry A, Gowda VRP, Torres RO, McNally KL, Serraj R. Variation in root system architecture and drought response in rice (Oryza sativa): Phenotyping of the OryzaSNP panel in rainfed lowland fields. Field Crop Res. 2011;120:205-14.

52. Cantarel BL, Korf I, Robb SMC, Parra G, Ross E, Moore B, et al. MAKER: an easy-to-use annotation pipeline designed for emerging model organism genomes. Genome Res. 2008;18:188-96.

53. Holt C, Yandell M. MAKER2: an annotation pipeline and genome-database management tool for second-generation genome projects. BMC Bioinformatics. 2011;12:491.

54. Choi JY, Purugganan MD. Evolutionary epigenomics of retrotransposonmediated methylation spreading in rice. Mol Biol Evol. 2018;35:365-82.

55. Sedlazeck FJ, Rescheneder P, Smolka M, Fang H, Nattestad M, von Haesele $A$, et al. Accurate detection of complex structural variations using singlemolecule sequencing. Nat Methods. 2018;15:461-8.

56. Alonge M, Soyk S, Ramakrishnan S, Wang X, Goodwin S, Sedlazeck FJ, et al. RaGOO: fast and accurate reference-guided scaffolding of draft genomes. Genome Biol. 2019;20:224.

57. Kawahara Y, de la Bastide M, Hamilton JP, Kanamori H, McCombie WR, Ouyang $S$, et al. Improvement of the Oryza sativa Nipponbare reference genome using next generation sequence and optical map data. Rice. 2013;6:4.

58. Sakai H, Lee SS, Tanaka T, Numa H, Kim J, Kawahara Y, et al. Rice annotation project database (RAP-DB): an integrative and interactive database for rice genomics. Plant Cell Physiol. 2013;54:e6.

59. Emms DM, Kelly S. OrthoFinder: solving fundamental biases in whole genome comparisons dramatically improves orthogroup inference accuracy. Genome Biol. 2015;16:157.

60. Nattestad M, Schatz MC. Assemblytics: a web analytics tool for the detection of variants from an assembly. Bioinformatics. 2016;32:3021-3.

61. Fuentes RR, Chebotarov D, Duitama J, Smith S, la Hoz JFD, Mohiyuddin M, et al. Structural variants in 3000 rice genomes. Genome Res. 2019;29:870-80

62. Shomura A, Izawa T, Ebana K, Ebitani T, Kanegae H, Konishi S, et al. Deletion in a gene associated with grain size increased yields during rice domestication. Nat Genet. 2008;40:1023-8.

63. Zhou Y, Zhu J, Li Z, Yi C, Liu J, Zhang H, et al. Deletion in a quantitative trait gene qPE9-1 associated with panicle erectness improves plant architecture during rice domestication. Genetics. 2009;183:315-24.

64. Lye ZN, Purugganan MD. Copy number variation in domestication. Trends Plant Sci. 2019;24:352-65.

65. Hu M, Lv S, Wu W, Fu Y, Liu F, Wang B, et al. The domestication of plant architecture in African rice. Plant J. 2018;94:661-9.

66. Wu Y, Zhao S, Li X, Zhang B, Jiang L, Tang Y, et al. Deletions linked to PROG1 gene participate in plant architecture domestication in Asian and African rice. Nat Commun. 2018:9:4157.

67. Li B, Zhang Y, Li J, Yao G, Pan H, Hu G, et al. Fine mapping of two additive effect genes for awn development in rice (Oryza sativa L.). PLoS One. 2016; 11:e0160792.

68. Hua L, Wang DR, Tan L, Fu Y, Liu F, Xiao L, et al. LABA1, a domestication gene associated with long, barbed awns in wild rice. Plant Cell. 2015;27:1875-88.

69. Zhu B-F, Si L, Wang Z, Zhou Y, Zhu J, Shangguan Y, et al. Genetic control of a transition from black to straw-white seed hull in rice domestication. Plant Physiol. 2011;155:1301-11.

70. Tan L, Li X, Liu F, Sun X, Li C, Zhu Z, et al. Control of a key transition from prostrate to erect growth in rice domestication. Nat Genet. 2008;40:1360-4.

71. Saitoh K, Onishi K, Mikami I, Thidar K, Sano Y. Allelic diversification at the C (OsC1) locus of wild and cultivated rice: nucleotide changes associated with phenotypes. Genetics. 2004;168:997-1007.

72. Li C, Zhou A, Sang T. Rice domestication by reducing shattering. Science. 2006;311:1936-9.

73. Fan C, Xing Y, Mao H, Lu T, Han B, Xu C, et al. GS3, a major QTL for grain length and weight and minor QTL for grain width and thickness in rice, encodes a putative transmembrane protein. Theor Appl Genet. 2006;112: 1164-71.

74. Choi JY, Zaidem M, Gutaker R, Dorph K, Singh RK, Purugganan MD. The complex geography of domestication of the African rice Oryza glaberrima. PLoS Genet. 2019;15:e1007414.

75. Kumar A, Bennetzen JL. Plant retrotransposons. Annu Rev Genet. 1999;33: 479-532.

76. Zuccolo A, Sebastian A, Talag J, Yu Y, Kim H, Collura K, et al. Transposable element distribution, abundance and role in genome size variation in the genus Oryza. BMC Evol Biol. 2007;7:152.
77. Lerat E. Identifying repeats and transposable elements in sequenced genomes: how to find your way through the dense forest of programs. Heredity. 2010;104:520-33.

78. Hoen DR, Hickey G, Bourque G, Casacuberta J, Cordaux R, Feschotte C, et al. A call for benchmarking transposable element annotation methods. Mob DNA. 2015;6:13.

79. Bennetzen JL. The contributions of retroelements to plant genome organization, function and evolution. Trends Microbiol. 1996;4:347-53.

80. Voytas DF, Ausubel FM. A copia-like transposable element family in Arabidopsis thaliana. Nature. 1988;336:242-4.

81. SanMiguel P, Gaut BS, Tikhonov A, Nakajima Y, Bennetzen JL. The paleontology of intergene retrotransposons of maize. Nat Genet. 1998;20:43-5.

82. Wicker T, Sabot F, Hua-Van A, Bennetzen JL, Capy P, Chalhoub B, et al. A unified classification system for eukaryotic transposable elements. Nat Rev Genet. 2007:8:973-82

83. Vitte C, Panaud O, Quesneville H. LTR retrotransposons in rice (Oryza sativa, L.): recent burst amplifications followed by rapid DNA loss. BMC Genomics. 2007;8:218.

84. Panaud O, Vitte C, Hivert J, Muzlak S, Talag J, Brar D, et al. Characterization of transposable elements in the genome of rice (Oryza sativa L.) using representational difference analysis (RDA). Mol Genet Genomics. 2002;268:113-21.

85. McCarthy EM, Liu J, Lizhi G, McDonald JF. Long terminal repeat retrotransposons of Oryza sativa. Genome biology. 2002;3:RESEARCH0053.

86. Carpentier M-C, Manfroi E, Wei F-J, Wu H-P, Lasserre E, Llauro C, et al. Retrotranspositional landscape of Asian rice revealed by 3000 genomes. Nat Commun. 2019;10. https://doi.org/10.1038/s41467-018-07974-5.

87. Zhang Q-J, Gao L-Z. Rapid and recent evolution of LTR retrotransposons drives rice genome evolution during the speciation of AA- genome Oryza species. G3. 2017:7(6):1875-85

88. Kumekawa N, Ohtsubo H, Horiuchi T, Ohtsubo E. Identification and characterization of novel retrotransposons of the gypsy type in rice. Mol Gen Genet. 1999:260:593-602.

89. Chaparro C, Guyot R, Zuccolo A, Piégu B, Panaud O. RetrOryza: a database of the rice LTR-retrotransposons. Nucleic Acids Res. 2007; 35(Database issue):D66-70.

90. Wang H, Vieira FG, Crawford JE, Chu C, Nielsen R. Asian wild rice is a hybrid swarm with extensive gene flow and feralization from domesticated rice. Genome Res. 2017;27:1029-38.

91. Li L-F, Li Y-L, Jia Y, Caicedo AL, Olsen KM. Signatures of adaptation in the weedy rice genome. Nat Genet. 2017:49:811-4.

92. Shimodaira $\mathrm{H}$. An approximately unbiased test of phylogenetic tree selection. Syst Biol. 2002;51:492-508.

93. Green RE, Krause J, Briggs AW, Maricic T, Stenzel U, Kircher M, et al. A draft sequence of the Neandertal genome. Science. 2010;328:710-22.

94. Durand EY, Patterson N, Reich D, Slatkin M. Testing for ancient admixture between closely related populations. Mol Biol Evol. 2011;28:2239-52.

95. Martin SH, Van Belleghem SM. Exploring evolutionary relationships across the genome using topology weighting. Genetics. 2017;206:429-38.

96. Gutenkunst RN, Hernandez RD, Williamson SH, Bustamante CD. Inferring the joint demographic history of multiple populations from multidimensional SNP frequency data. PLoS Genet. 2009:5:e1000695.

97. Raj A, Stephens M, Pritchard JK. fastSTRUCTURE: variational inference of population structure in large SNP data sets. Genetics. 2014;197:573-89.

98. Michael TP, Jupe F, Bemm F, Motley ST, Sandoval JP, Lanz C, et al. High contiguity Arabidopsis thaliana genome assembly with a single nanopore flow cell. Nat Commun. 2018:9:541.

99. Schmidt MH, Vogel A, Denton AK, Istace B, Wormit A, van de Geest $H$, et al. De novo assembly of a new Solanum pennellii accession using nanopore sequencing. Plant Cell. 2017;29(10):2336-48.

100. Belser C, Istace B, Denis E, Dubarry M, Baurens F-C, Falentin C, et al. Chromosome-scale assemblies of plant genomes using nanopore long reads and optical maps. Nature Plants. 2018:4:879.

101. Howe K, Wood JM. Using optical mapping data for the improvement of vertebrate genome assemblies. GigaScience. 2015;4:10

102. Goodwin S, McPherson JD, McCombie WR. Coming of age: ten years of next-generation sequencing technologies. Nat Rev Genet. 2016;17:333-51.

103. Udall JA, Dawe RK. Is it ordered correctly? Validating genome assemblies by optical mapping. Plant Cell. 2018;30:7-14.

104. Sedlazeck FJ, Lee H, Darby CA, Schatz MC. Piercing the dark matter: bioinformatics of long-range sequencing and mapping. Nat Rev Genet. 2018;19:329-46. 
105. Wendel JF, Jackson SA, Meyers BC, Wing RA. Evolution of plant genome architecture. Genome Biol. 2016;17:37.

106. vonHoldt BM, Takuno S, Gaut BS. Recent retrotransposon insertions are methylated and phylogenetically clustered in japonica rice (Oryza sativa spp. japonica). Mol Biol Evol. 2012;29:3193-203.

107. Baucom RS, Estill JC, Leebens-Mack J, Bennetzen JL. Natural selection on gene function drives the evolution of LTR retrotransposon families in the rice genome. Genome Res. 2009;19:243-54.

108. Naito K, Zhang F, Tsukiyama T, Saito H, Hancock CN, Richardson AO, et al. Unexpected consequences of a sudden and massive transposon amplification on rice gene expression. Nature. 2009;461:1130-4.

109. Civáň P, Ali S, Batista-Navarro R, Drosou K, Ihejieto C, Chakraborty D, et al. Origin of the aromatic group of cultivated rice (Oryza sativa $\mathrm{L}$.) traced to the Indian subcontinent. Genome Biol Evol. 2019;11:832-43.

110. Santos JD, Chebotarov D, McNally KL, Bartholomé J, Droc G, Billot C, et al. Fine scale genomic signals of admixture and alien introgression among Asian rice landraces. Genome Biol Evol. 2019;11:1358-73.

111. Molina J, Sikora M, Garud N, Flowers JM, Rubinstein S, Reynolds A, et al. Molecular evidence for a single evolutionary origin of domesticated rice. Proc Natl Acad Sci U S A. 2011;108:8351-6.

112. Fuller DQ. Finding plant domestication in the Indian subcontinent. Curr Anthropol. 2011;52:S347-62.

113. Filiault DL, Ballerini ES, Mandáková T, Aköz G, Derieg NJ, Schmutz J, et al. The Aquilegia genome provides insight into adaptive radiation and reveals an extraordinarily polymorphic chromosome with a unique history. eLife. 2018; :e36426.

114. Liakat Ali M, McClung AM, Jia MH, Kimball JA, McCouch SR, Georgia CE. A rice diversity panel evaluated for genetic and agro-morphological diversity between subpopulations and its geographic distribution. Crop Sci. 2011;51:2021-35.

115. Travis AJ, Norton GJ, Datta S, Sarma R, Dasgupta T, Savio FL, et al. Assessing the genetic diversity of rice originating from Bangladesh, Assam and West Bengal. Rice (N Y). 2015;8:35.

116. Glaszmann JC. Geographic pattern of variation among Asian native rice cultivars (Onzza sativa L.) based on fifteen isozyme loci. Genome. 1988;30:782-92.

117. Myint KM, Courtois B, Risterucci A-M, Frouin J, Soe K, Thet KM, et al. Specific patterns of genetic diversity among aromatic rice varieties in Myanmar. Rice (N Y). 2012;5. https://doi.org/10.1186/1939-8433-5-20.

118. Zhang H-B, Zhao X, Ding X, Paterson AH, Wing RA. Preparation of megabase-size DNA from plant nuclei. Plant J. 1995;7:175-84.

119. Koren S, Walenz BP, Berlin K, Miller JR, Bergman NH, Phillippy AM. Canu: scalable and accurate long-read assembly via adaptive $\mathrm{k}$-mer weighting and repeat separation. Genome Res. 2017;27:722-36.

120. Kolmogorov M, Yuan J, Lin Y, Pevzner PA. Assembly of long, error-prone reads using repeat graphs. Nat Biotechnol. 2019;37:540

121. Vaser R, Sović I, Nagarajan N, Šikić M. Fast and accurate de novo genome assembly from long uncorrected reads. Genome Res. 2017;27:737-46.

122. Li H. Aligning sequence reads, clone sequences and assembly contigs with BWA-MEM. arXiv. 2013:1303.3997v2.

123. Walker BJ, Abeel T, Shea T, Priest M, Abouelliel A, Sakthikumar S, et al. Pilon: an integrated tool for comprehensive microbial variant detection and genome assembly improvement. PLoS One. 2014:9:e112963.

124. Li H. Minimap2: pairwise alignment for nucleotide sequences. Bioinformatics. 2018:34:3094-100

125. Simão FA, Waterhouse RM, loannidis $P$, Kriventseva EV, Zdobnov EM. BUSCO: assessing genome assembly and annotation completeness with single-copy orthologs. Bioinformatics. 2015;31:3210-2.

126. Cabanettes F, Klopp C. D-GENIES: dot plot large genomes in an interactive, efficient and simple way. PeerJ. 2018;6. https://doi.org/10.7717/peerj.4958.

127. Marçais G, Delcher AL, Phillippy AM, Coston R, Salzberg SL, Zimin A. MUMmer4: a fast and versatile genome alignment system. PLoS Comput Biol. 2018;14:e1005944.

128. Korf I. Gene finding in novel genomes. BMC Bioinformatics. 2004;5:59.

129. Stanke M, Diekhans M, Baertsch R, Haussler D. Using native and syntenically mapped CDNA alignments to improve de novo gene finding. Bioinformatics. 2008;24:637-44.

130. Conway JR, Lex A, Gehlenborg N, Hancock J. UpSetR: an R package for the visualization of intersecting sets and their properties. Bioinformatics. 2017; 33:2938-40.

131. Tian T, Liu Y, Yan H, You Q, Yi X, Du Z, et al. agriGO v2.0: a GO analysis toolkit for the agricultural community, 2017 update. Nucleic Acids Res. 2017; 45(Web Server issue):W122-9.
132. Huerta-Cepas J, Forslund K, Coelho LP, Szklarczyk D, Jensen LJ, von Mering $C$, et al. Fast genome-wide functional annotation through orthology assignment by eggNOG-Mapper. Mol Biol Evol. 2017;34:2115-22.

133. Falcon S, Gentleman R. Using GOstats to test gene lists for GO term association. Bioinformatics. 2007;23:257-8.

134. Ellinghaus D, Kurtz S, Willhoeft U. LTRharvest, an efficient and flexible software for de novo detection of LTR retrotransposons. BMC bioinformatics. 2008;9:18.

135. Copetti D, Zhang J, El Baidouri M, Gao D, Wang J, Barghini E, et al. RiTE database: a resource database for genus-wide rice genomics and evolutionary biology. BMC Genomics. 2015;16:538.

136. Camacho C, Coulouris G, Avagyan V, Ma N, Papadopoulos J, Bealer K, et al. BLAST+: architecture and applications. BMC Bioinformatics. 2009;10:421.

137. Katoh K, Standley DM. MAFFT multiple sequence alignment software version 7 : improvements in performance and usability. Mol Biol Evol. 2013;30:772-80

138. Yang Z. PAML 4: phylogenetic analysis by maximum likelihood. Mol Biol Evol. 2007;24:1586-91.

139. Kimura M. A simple method for estimating evolutionary rates of base substitutions through comparative studies of nucleotide sequences. J Mol Evol. 1980;16:111-20

140. Ma J, Bennetzen JL. Rapid recent growth and divergence of rice nuclear genomes. Proc Natl Acad Sci U S A. 2004;101:12404-10.

141. Quinlan AR, Hall IM. BEDTools: a flexible suite of utilities for comparing genomic features. Bioinformatics. 2010;26:841-2.

142. Haudry A, Platts AE, Vello E, Hoen DR, Leclerca M, Williamson RJ, et al. An atlas of over 90,000 conserved noncoding sequences provides insight into crucifer regulatory regions. Nat Genet. 2013;45:891-8.

143. Harris RS. Improved pairwise alignment of genomic DNA. State College: PhD Thesis, The Pennsylvania State University; 2007.

144. Kent WJ, Baertsch R, Hinrichs A, Miller W, Haussler D. Evolution's cauldron: duplication, deletion, and rearrangement in the mouse and human genomes. Proc Natl Acad Sci U S A. 2003:100:11484-9.

145. Blanchette M, Kent WJ, Riemer C, Elnitski L, Smit AFA, Roskin KM, et al. Aligning multiple genomic sequences with the threaded blockset aligner. Genome Res. 2004;14:708-15.

146. Hubisz MJ, Pollard KS, Siepel A. PHAST and RPHAST: phylogenetic analysis with space/time models. Brief Bioinformatics. 2011;12:41-51.

147. Stamatakis A. RAxML version 8: a tool for phylogenetic analysis and postanalysis of large phylogenies. Bioinformatics. 2014;30:1312-3.

148. Martin SH, Jiggins CD. Interpreting the genomic landscape of introgression. Curr Opin Genet Dev. 2017;47:69-74.

149. Goldman N, Anderson JP, Rodrigo AG, Olmstead R. Likelihood-based tests of topologies in phylogenetics. Syst Biol. 2000;49:652-70.

150. Shimodaira H, Hasegawa M. CONSEL: for assessing the confidence of phylogenetic tree selection. Bioinformatics. 2001;17:1246-7.

151. Van der Auwera GA, Carneiro MO, Hartl C, Poplin R, del Angel G, LevyMoonshine A, et al. From FastQ data to high-confidence variant calls: the genome analysis toolkit best practices pipeline. In: Current protocols in bioinformatics. Hoboken: Wiley; 2013. p. 11.10.1-11.10.33. https://doi.org/10. 1002/0471250953.bi1110s43.

152. Danecek P, Auton A, Abecasis G, Albers CA, Banks E, DePristo MA, et al. The variant call format and VCFtools. Bioinformatics. 2011;27:2156-8.

153. Browning BL, Browning SR. Genotype imputation with millions of reference samples. Am J Hum Genet. 2016;98:116-26.

154. Purcell S, Neale B, Todd-Brown K, Thomas L, Ferreira MAR, Bender D, et al. PLINK: a tool set for whole-genome association and population-based linkage analyses. Am J Hum Genet. 2007;81:559-75.

155. Freedman AH, Gronau I, Schweizer RM, Ortega-Del Vecchyo D, Han E, Silva PM, et al. Genome sequencing highlights the dynamic early history of dogs. PLoS Genet. 2014;10:e1004016.

156. Lefort V, Desper R, Gascuel O. FastME 2.0: a comprehensive, accurate, and fast distance-based phylogeny inference program. Mol Biol Evol. 2015;32: 2798-800.

157. Xu X, Liu X, Ge S, Jensen JD, Hu F, Li X, et al. Resequencing 50 accessions of cultivated and wild rice yields markers for identifying agronomically important genes. Nat Biotechnol. 2012;30:105-11.

158. Pickrell JK, Pritchard JK. Inference of population splits and mixtures from genome-wide allele frequency data. PLoS Genet. 2012;8:e1002967.

159. Choi JY, Zye ZN, Groen SC, Dai X, Rughani P, Zaaijer S, et al. Nanopore sequencing and assembly of Basmati rice. European Nucleotide Archive. (2019). https://www.ebi.ac.uk/ena/browser/view/PRJEB28274. 
160. Choi JY, Zye ZN, Groen SC, Dai X, Rughani P, Zaaijer S, et al. Nanopore sequencing and assembly of Sadri rice. European Nucleotide Archive. (2019). https://www.ebi.ac.uk/ena/browser/view/PRJEB32431.

161. Choi JY, Zye ZN, Groen SC, Dai X, Rughani P, Zaaijer S, et al. Whole genome re-sequencing of a mini-core set of Oryza sativa landraces for studies of salinity tolerance in rice. European Nucleotide Archive. (2019). https://www. ebi.ac.uk/ena/browser/view/PRJNA422249.

162. Choi JY, Zye ZN, Groen SC, Dai X, Rughani P, Zaaijer S, et al. Illumina sequencing of Oryza sativa subpopulation/variety. European Nucleotide Archive. (2019). https://www.ebi.ac.uk/ena/browser/view/PRJNA557122.

163. Choi JY, Zye ZN, Groen SC, Dai X, Rughani P, Zaaijer S, et al. Dataset for "Nanopore-based genome assembly and the evolutionary genomics of basmati rice". Zenodo. 2019; https://doi.org/10.5281/zenodo.3355330.

\section{Publisher's Note}

Springer Nature remains neutral with regard to jurisdictional claims in published maps and institutional affiliations.

Ready to submit your research? Choose BMC and benefit from:

- fast, convenient online submission

- thorough peer review by experienced researchers in your field

- rapid publication on acceptance

- support for research data, including large and complex data types

- gold Open Access which fosters wider collaboration and increased citations

- maximum visibility for your research: over $100 \mathrm{M}$ website views per year

At $\mathrm{BMC}$, research is always in progress.

Learn more biomedcentral.com/submissions 